\title{
Verhandlungen der gynäkologischen Section der 59. Versammlung deutscher Naturforseher und Aerzte in Berlin. 1886.
}

\author{
Berichtet \\ von \\ Doc. Dr. Th. Wyder, \\ Berlin.
}

I. Sitzungstag: Montig, den 20. September.

A. Demonstrationen in der kgl. Frauenklinik, Artilleriestrasse.

Kehrer (Heidelberg) demonstrirt eine Nachgeburt von Zwillingen mit einfachem Chorion und Amnion. Die Nabelschnüre sind mehrfach mit einander verschlungen und enthalten wahre Knoten. Die Insertionen sind auf $3 \mathrm{~cm}$ genähert. Beide Kinder leben und wogen 1800 resp. 1900 g. Das zweite Kind war leicht asphyktisch; seine Geburt war ferner durch Armvorfall und Hydramnios erschwert. Beide Zwillinge wurden in Schädellage geboren.

Neugebauer (Warschau) weist vor:

a) ein seltenes Präparat von Foetus amorphus;

b) das in seiner letzten Arbeit in diesem Archiv beschriebene Präparat von Wirbelschiebung;

c) eine Wirbelsäule mit congenital bilateraler Interarticularpseudarthrose des dritten bis fünften Lendenwirbels.

M. Hofmeier (Berlin) zeigt:

1) eine Serie von Tuben- und Tuboovarialpräparaten: Je 3 Fälle von Hydro- und Pyosalpinx; 1 Fall von Tubenschwangerschaft aus dem zweiten Monate; 1 Fall von Tuboovarialschwangerschaft mit ausgetragenem Kinde. Die zum Theil faustgrossen Tumoren waren meist eingehüllt in allseitige Adhäsionen und infolge dessen recht schwierig zu entfernen. In 
einem Falle platzte die Pyosalpinx und entleerte ihren stinkenden Eiter in die Bauchhöhle. Mit Ausnahme des letzten Falles, in welchem eine vollkommene Verjauchung mit Gasbildung eingetreten, genasen sämmtliche Patientinnen;

2) ein Präparat von Oophoritis chronica, welche hohes Fieber und starke Schmerzen verursacht hatte und wo durch operative Entfernung völlige Genesung geschaffen wurde;

3) eine Serie von 4 Uteri, welche von Eklamptischen stammen, die in den ersten 36 Stunden verstorben waren. Behufs des Studiums der Gefässvertheilung am unteren Abschnitte und am Gebärmutterkörper wurden die Gefässe an den herausgenommenen Präparaten injicirt und präparirt. Es zeigen die Präparate, dass, abgesehen von einem grösseren, regelmässig an das obere Scheidengewölbe gehenden Ast, die Uterinae fast ganz frei und unverästelt an der Seitenkante des Uterus herauflaufen und die nächsten grösseren Verzweigungen erst weit über der Stelle des Contractionsringes in die Uterussubstanz eindringen. Dieselben Verhältnisse zeigt auch ein Uterus mit Placenta praevia an dem Ende der Schwangerschaft. Es war hier bei einer schon sterbenden Frau die combinirte Wendung gemacht, wegen des bereits festgestellten Todes des Kindes aber die Extraction nicht ausgeführt worden. Der Uterus wurde mit Inhalt herausgenommen, injicirt, gehärtet und aufgeschnitten. Der Uterus zeigt zunächst in ausserordentlich schöner Weise die Cervix, den inneren Muttermund; die Stelle des unteren Uterinsegmentes stark entwickelt. Die Placenta war noch bis zum inneren Muttermunde fest anhaftend. Nur an einer Stelle ist sie infolge der combinirten Wendung etwas gelöst. Auch hier giebt an der Seite, wo die Placenta anliegt, die Utexina nur zwei mittelgrosse Zweige an die Substanz des Uterus ab, um erst wieder weiter oben in den Uterus selbst einzutreten.

Czempin (Berlin):

1) Demonstration zweier Präparate von Extrauterinschwangerschaft. Im ersten Falle bestand eine viermonatliche Schwangerschaft. Hinter dem Uterus, der in Bezug auf Grösse diesem Zeitpunkte entsprach, fühlte man einen prallelastischen, überkindskopfgrossen Tumor, der mit Uterus und Beckenboden verwachsen für einen Ovarialtumor gehalten wurde. Mit Rücksicht auf die angenommene intra uterine Gravidität und des am Ende derselben durch den Tumor sicher in Aussicht 
310 Verhandlungen der gynäkologischen Section der 59. Versammlung

stehenden Geburtshindernisses wurde die Laparatomie vorgenommen. Infolge der festen und allseitigen Verwachsungen war die Exstirpation der Geschwulst sehr schwierig; dieselbe platzte dabei und traten Fötus und Placenta durch den Riss aus. Wahrscheinlich handelte es sich um eine Tubarschwangerschaft. - Im zweiten Falle lag ein Lithopädion, schon seit 12 Jahren getragen, vor. Dasselbe entstammt einem wahrscheinlich im vierten Monate geborstenen Eileiterfruchtsacke. Nach dem Austritte des Fötus wurde derselbe anscheinend bis ans Ende der Schwangerschaft von der Insertionsstelle des Eies aus ernährt.

2) Demonstration eines Cystosarkoma ovarii dextri. Das Präparat, kindskopfgross, entstammt einer 37jährigen Nullipara und verursachte starke Metrorrhagien. Vor vier Monaten wurde der Tumor entfernt und blieb bis jetzt Patientin von Rückfall verschont.

Orthmann (Berlin): 8 Präparate, herrührend von Salpingotomien, ausgeführt von Martin. Viermal bestand Salpingitis catarrhalis, viermal Pyosalpinx. Die Untersuchung auf Mikroorganismen fiel negativ aus.

Derselbe demonstrirt ferner Zeichnungen von Längsand Querschnitten durch beide Enden normaler Tuben, welche namentlich das Verhalten der Tubarmucosa veranschaulichen.

Veit (Berlin) zeigt der Versammlung zwei Präparate:

1) ein subseröses Uterusmyom, nierenförmig, mit breitem weichen Stiele versehen, operativ entfernt;

2) ein überapfelgrosses Papillom der Blase. Die Gegenwart des Tumors wurde nach Dilatation der Urethra festgestellt; von einer Entfernung durch die Scheide musste wegen der Enge derselben abgesehen und die Sectio alta gewählt werden. Trotz der sehr fettreichen Bauchdecken war die Operation leicht und gelangte man, ohne das Peritoneum zu verletzen, zum Stiele der Geschwulst.

Winter (Berlin) legt vor :

a) Den Uterus einer an Eklampsie verstorbenen Frau, an welcher nach dem Tode die Sectio caesarea vorgenommen worden war. Wehen waren bei der am Ende der Schwangerschaft befindlichen Frau noch nicht eingetreten. Die Cervix ist 
der ganzen Länge nach erhalten, 4,3 cm lang. Die Eihäute sind bis an den inneren Muttermund anhaftend. Die Placenta überwölbt die durch postmortale Contraction verkleinerte Placentarstelle nach allen Seiten.

b) Uterus einer im siebenten Monat an Eklampsie Verstorbenen, bei welcher ebenfalls nach dem Tode der Kaiserschnitt vorgenommen wurde. Das untere Uterinsegment ist verdünnt; der Contractionsring an der Ansatzstelle des Peritoneum gebildet; die Cervix ist hinten vollkommen verstrichen, vorn stark verkürzt. Die Grenze der Cervicalschleimhaut gegen das untere Uterinsegment ist an den Krypten deutlich sichtbar.

c) 4 Placenten mit Blutungen und vorzeitiger Lösung bei Nephritis. In einem Falle war die Placenta zur Hälfte gelöst, in zwei Fällen bestanden nur haselnuss- bezw. wallnussgrosse Blutungen, im vierten Falle sass die Quelle der Blutung in der Placenta, welche durch dieselbe zur Hälfte zertrümmert war. Drei Mal bestand Schwangerschaftsnephritis, ein Mal die interstitielle Form.

Stratz (Berlin) zeigt

1) die Originale der in Gemeinschaft mit Schroeder veröfentlichten Gefrierdurchschnitte durch eine Kreissende und eine Wöchnerin;

2) einen Gefrierdurchschnitt durch eine Wöchnerin.

B. Vorträge in der Aula der Universität.

1) Olshausen (Halle): Ueber ventrale Operationen bei Lageanomalien.

Annäherung der Cornua uteri an die Bauchdecken und Fixam tion daselbst empfiehlt Olshausen als ein einfaches Operationsverfahren, welches zunächst bei Laparatomien wegen Tumoren, complicirt durch schwere Störung verursachende, auf andere Weise nicht $\mathrm{zu}$ beseitigende Lageanomalien, in Anwendung kommen soll, welches aber auch da gerechtfertigt erscheine und in Betracht zu ziehen sei, wo eine Deviation des Uterus alle in bestehe, die starke, jeder anderen Therapie trotzende Beschwerden im Gefolge habe.

Besprechung.

Frank (Cöln) ist mit diesem Operationsverfahren nicht einverstanden, besonders wenn es sich um angeheftete Retroflexionen Archiv f. Gynäkologie. Bd. XXIX. Heft 2. 


\section{Verhandilungen der gynäkologisehen Section der 59. Versammlung}

handelt. Bei Anlass von Castrationen sah er vor etwa 6 Jahren Bardenheuer die Ligamenta rotunda und Tuben durch die Bauchdecken ziehen und hier befestigen. Die Stümpfe, welche sicher anheilten, haben sich aber in der Folgezeit wieder gedehnt, der Uterus nahm seine regelwidrige Lage wieder ein, verursachte grosse Beschwerden und musste später exstirpirt werden.

Für so verzweifelte Fälle empfiehlt Frank dann, wenn das Klimakterium bereits überschritten sei, die extraperitoneale Ausschälung und Abtragung des Uterus. In einem Falle, wo von anderer Seite eine Frau castrirt worden war, wo der Uterus mit Blase verwachsen und die Patientin infolge dessen unvermögend war, den Urin spontan zu lassen, hat er auf diese Weise die Frau völlig hergestellt.

Hennig (Leipzig) sympathisirt mit dem Vorschlage Ols hausen's auf Grund einer vor 6 Jahren gemachten Beobachtung. Im Anschluss an eine wegen Metrorrhagien bei chronisch entzündetem; vergrössertem, retroflectirtem Uterus vorgenommenen Castration befestigte er nach modificirter Koeberlé'scher Methode den letzteren an den Bauchdecken und schuf so völlige Genesung.

Kaltenbach (Giessen) erinnert daran, dass von Czerny ein dem Olshause n'schen ähnliches Verfahren bei befestigten Retroflexionen angegeben worden sei. Dabei seien aber die Uterusanhänge mit entfernt worden und sei nach seinem Dafürhalten die eingetretene Heilung auf letzteres Verfahren zurückzuführen, weil bei befestigter Retroflexion der Peritonitis pelvica gewöhnlich eine Erkrankung der Tuben und Ovarien zu Grunde liege und ohne die Entfernung dieses Ausgangspunktes der Erkrankung eine vollkommene Beseitigung der Beschwerden wohl kaum zu erwarten sei. Für diese Anschauung spreche auch eine Beobachtung, wonach bei einer Patientin nach Entfernung der Uterusadnexa alle Beschwerden sofort aufhörten, obschon der Uterus in seiner festen Retroflexionsstellung belassen wurde.

2) Schatz (Rostock): Ueber Physiologie und physiologische Behandlung der Nachgeburtsperiode.

Sowohl die von Baudelocque und B. Schultze einerseits, als von $D$ unean andererseits beschriebene Art der Geburt der Placenta kommt vor und glaubt Redner für beide Arten eine richtige Erklärung gefunden zu haben in der von oben nach unten fortschreitenden peristaltischen Contraction des Uterus. 
Eine im Fundus sitzende Placenta wird immer mit umgestïlpten Eihäuten geboren werden.

Eine seitlich inserirte wird bei genügend kräftiger Contraction des Uterus kurz vor oder nach der Ausstossung des Kindes in der Regel in folgender Weise gelöst:

„Durch die Contraction des Uterusgrundes wird zuerst der obere Rand der Placenta durch Flächenreduction losgelöst und da das Lumen des Uterusgrundes dabei zugleich sehr stark verkleinert wird, nach der zunächst tieferen Utoruszone eingerollt. Zieht sich nunmehr diese Zone ebenfalls stark zusammen, so wird auch der an ihr befindliche Theil der Placenta gelöst, und da er nicht nach dem contrahirten und verengten Uterusgrund ausweichen kann, zugleich mit dem schon eingerollten oberen Rande der Placenta tiefer getrieben. Auf diese Weise wird mit fortschreitender Peristaltik die ganze Placenta allmälig von oben nach unten ganz ähnlich eingerollt und mit dem ursprünglich oberen Rande zuerst geboren, wie der Geburtshelfer dies bei der künstlichen Placentarlösung ausfïhren soll. Natürlich ist es nicht immer ganz genau der obere Rand der Placenta, welcher im Muttermunde erscheint. Dieser muss die anhaftenden Eihäute nach sich ziehen und wird dadurch, wenn er einigermaassen dünn ist, mehr weniger zurückgehalten, so dass dann nur ein ihm naher Theil der fötalen Placentaroberfläche zuerst durch den Muttermund tritt. Bei solcher idealen, d.h. durch eine einzige Wehe erzeugten Loslösung und Ausstossung der Placenta kommt gar keine Blutung zu Stande; denn so lange die Placenta noch haftet, blutet der Uterus auch ohne Contraction nicht. Jeder Theil des Uterus aber, welcher sich so stark contrahirt, dass dadurch der entsprechende Theil der Placenta losgelöst wird, ist durch Contraction blutleer. Blutungen erfolgen dadurch, dass die Ablösung sehr häufig nicht mit einer Uteruscontraction zu Stande kommt, so dass in der betreffenden Wehenpause die Grenzstellen, besonders zwischen gelöster und noch adhärenter Placenta, rom Uterus aus Blut ergiessen. Der sog. retroplacentare Bluterguss ist also mindestens überflüssig. Er ist nur so häufig, weil die erste oder die ersten Wehen nach der Ausstossung des Kindes recht häufig durch zu schnelle Entleerung des Uterus unvollkommen sind.

Durchaus nicht alle Placenten aber werden so geboren, wie Baudelocque beschreibt. Duncan bildet bei Darlegung seines 
314 Verhandlungen der gynäkologischen Section der 59. Versammlung

Mechanismus die Placenta reichlich dick ab. Solche compacte Placenten werden in Wahrheit recht häufig mit dem unteren Rande zuerst geboren. Wenn nämlich der obere Theil der Placenta sehr fest und dick ist, dass er bei der Loslösung aus dem verkleinerten Uterusgrund nicht durch Einrollung nach dem Uteruslumen gelangen kann, so wird er ohne Einrollung tiefer gedrängt und drängt die unter ihm liegenden tieferen Zonen der Placenta ebenfalls tiefer, so dass diese durch Verschiebung schon früher gelöst werden können, als dies durch die Flächenreduction geschehen wäre. Zwischen dem ersten Mechanismus und dem letzten giebt es vermöge der verschiedenen Lageform und Festigkeit der Placenta alle möglichen Uebergänge."

In Nutzanwendung des Gesagten auf die Behandlung der Nachgeburtsperiode hebt Schatz hervor, dass wir hier ebenso individualisiren müssen wie bei anderen therapeutischen Maassnahmen. Unter Hervorhebung der bedeutenden Vortheile des Credé'schen Handgriffes weist Schatz darauf hin, dass demselben, selbst wenn er richtig ausgeübt werde, doch einige Gefahren ankleben, unter welchen in erster Linie das leichte $\mathrm{Zu}$ rückbleiben von Eihautresten zu erwähnen ist. Wird aber der Handgriff zu unrichtiger Zeit und in falscher Weise geübt, so straft sich dies öfters durch Retention von Placentarresten. Bei genügender Kenntniss des durchaus nicht uninteressanten Mechanismus der Nachgeburtsperiode und richtiger Individualisirung wird der Arzt im Stande sein, hier durch Abwarten, dort durch Eingreifen segensreich zu wirken; für ihn darf keine Methode die allein zu übende sein.

Besprechung.

Kehrer (Heidelberg): Da man weitaus in der Mehrzahl der Fälle nach der Geburt des Kindes die Placenta im Uterus gelöst findet und man ferner peristaltische Contractionen der Gebärmutter während der Austreibung des Kindes nicht fühlen kann, so ist doch wohl auch die Erklärung von Schatz über die Lösung dex Placenta nicht zutreffend. Höchstens kann auf diese Weise die Faltung, die Depression des oberen Randes, die Austreibung der Placenta in der dritten Periode erfolgen, wenn anders sich nicht die bekannte Umdrehung mit Bildung des retroplacentaren Blutergusses nach der Schultze'schen Theorie erklärte:

Schultze (Jena): Die Lösung der Placenta wird normaliter nicht von den Nachwehen besorgt, sondern sie kommt zu Stande 
durch die dauernde Verkürzung des Uterus während der Austreibung des Kindes. Infolge dessen wird die Placenta in der Mitte abgehoben; es tritt ein retroplacentarer Bluterguss ein, welcher dieselbe in die Uterushöhle hineindrängt. Die Umstïlpung bleibt aus in jenen Fällen, wo bei der Verkleinerung des Uterus der Fruchtkuchen gleich vollständig gelöst wird und nur ein minimaler Bluterguss erfolgt; ferner da, wo der retroplacentare Bluterguss zwischen Uterus und Eihäuten Abfluss findet. Auch die neuesten Beobachtungen Sch r o eder's bestätigen dieAnschauungen Schultze's.

Schroeder (Berlin) will ebenfalls die Erklärung der Loslösung der Placenta durch Peristaltik nur ausnahmsweise zulassen; wie und an welcher Stelle die Placenta zuerst sich löst, hängt besonders von dem anatomischen Zusammenhang zwischen letzterer und dem Uterus ab und von den etwas wechselnden Contractionen der Gebärmutter, welche an der Insertionsstelle des Uterus etwas gehindert sind.

Schatz führt gegen Kehrer an, dass die Placenta nach der Ausstossung des Kindes durchaus nicht immer gelöst ist. Sie ist es nur dann, wenn die Entleerung des Uterus genügend langsam erfolgt, beispielsweise, wenn nach der Geburt des Kopfes die Ausstossung des Rumpfes erst in der nächsten oder übernächsten Wehe erfolgt. Die Peristaltik des Uterus ist bei gefülltem Zustande des Uterus ebenso vorhanden wie bei entleertem, nur ist sie dort weniger leicht erkennbar. Die Erklärung von Schultze trifft für alle jene Fälle nicht zu, wo der obere Placentarrand zuerst geboren wird.

Gegenüber Schroeder hebt Redner hervor, dass die Placentarstelle nicht gelähmt ist, wie es vielleicht nach den Sectionsbefunden scheinen möchte. Es kann sich dieselbe bei noch vorhandener Placenta nicht ebenso ungehindert contrahiren wie die übrige Gebärmutterwand und bleibt deshalb bei mangelhafter Wehe grösser und dünner.

3) Rydygier (Culm): Zur Technik der Operation der Blasenscheidenfisteln.

Rydygier berichtet über 21 Blasenscheidenfisteloperationen, von denen nur ein Mal keine Heilung eintrat bei einer Patientin, welche wegen Psychose aus der Behandlung entlassen werden musste. Fast immer ist er mit der Simon'schen Methode aus- 
316 Verhandlungen der gynäkologischen Section der 59. Versammlung

gekommen. Nur zwei Mal liess ihn dieselbe im Stiche. Im ersten Falle, wo anderwärts nach zweimaliger vergeblicher Operation die quere Obliteration der Scheide vorgeschlagen worden war, gelang es Rydygier, die Fistel unmittelbar zu schliessen, nachdem er die Portio vaginalis seitlich hoch hinauf getrennt hatte und dieselbe auf diese Weise stark beweglich geworden war. Es wurde nun die angefrischte vordere Muttermundslippe in den Defect eingenäht. Zur Verringerung der Spannung werden Bindenzügel, durch die hintere Lippe gelegt, straff angezogen und an der Innenfläche der Oberschenkel durch Naht befestigt. So gelang es vor der Hand, den grössten Theil der Fistel zur Heilung zu bringen und schufen zwei Nachoperationen vollständigen Verschluss.

In einem zweiten Falle, wo die Fistel noch grösser war, wo auch ein Endresultat noch aussteht, will Rydygier den Defect schliessen durch einen gestielten Lappen aus der hinteren Vaginalwand. Ein grosses Instrumentarium ist bei Blasenscheidenfisteln nicht nöthig.

Mit Schroeder' ist er der Ansicht, dass Blasenscheidenfisteln häufiger durch den Druck der sich verzögernden Geburt als durch geburtshülfliche Instrumente zu Stande kommen. In Bezug auf die Behinderung der Conceptionsfähigkeit stimmt er Winckel zu, welcher glaubt, dass Urinfisteln als solche die Conception hindern ohne Mitwirkung von Veränderungen an der Gebärmutter.

4) Fränkel, E. (Breslau): Ueber die Erfolge der mechanischen Behandlung der häufigsten Formen ron Retrodeviation des Uterus.

Unter 5180 rein gynäkologischen Fällen aus Privatpraxis und Poliklinik in den Jahren 1882-85 behandelte Fränkel 936 Retrodeviationen $(18,07 \%)$. Unter diesen betrafen

417 oder 13,8\% Patienten aus der Privatpraxis

und $519,24,9 \% \quad, \quad, \quad$, Poliklinik.

Im Ganzen handelte es sich um 645 Retroflexionen und 291 Versionen, womit die Behauptung von Bantock, dass Retroversionen überwiegend häufig seien, widerlegt erscheint.

Da die poliklinischen Ziffern für die Entscheidung der dauernden Heilerfolge bei Retrodeviationen unbrauchbar erscheinen, so hat er sich auf die Resultate der Privatpraxis beschränkt, welche 
sich, wie oben erwähnt, auf ein Material von 417 Fällen beziehen. Von diesen gehen $a b$ :

1) 26 Fälle, die zum Theil völlig symptomlos, keiner Behandlung bedurften;

2) 58 Fälle, wo wegen Irreponibilität' keine mechanische Behandlung möglich war;

3) 39 Fälle aus der Behandlung geblieben (durch feste Adhäsionen).

Mechanisch behandelt wurden also 294 Fälle oder 70,5\% der in der Privatpraxis beobachteten Patientinnen.

Wo es immer anging, hat Fränkel manuell reponirt; nur $23 \mathrm{Mal} \mathrm{kam} \mathrm{die} \mathrm{Sonde} \mathrm{in} \mathrm{Anwendung;} 47 \mathrm{Mal}$ war Chloroformnarkose nöthig. Der aufgerichtete Uterus wurde seit 1882 fast ausschliesslich durch Thomas'sche Pessare fixirt und rühmt Fränkel an denselben hauptsächlich, dass sie den einmal reponirten Uterus vollkommen sicher in Normallage halten, dass sie $1 / 2-1$ Jahr lang ohne Wechsel getragen werden können und sehr sauber sind. Die Möglichkeit der Cohabitation und Conception ist dabei nicht ausgeschlossen und beqbachtete er letztere bei 40 Frauen während der Pessarbehandlung, bei einzelnen zwei- bis dreimalige.

Ein weiter angeführter Vortheil der Pessare besteht in deren ausreichenden Biegsamkeit und Formverschiedenheit.

Geburt und. Wochenbett heilen nach den Erfahrungen von Fränkel die Retrodeviationen fast nie. Dagegen bestätigt er die Möglichkeit der definitiven Heilung durch Thomas'sche Pessare. Unter 294 mechanisch behandelten Fällen hat er 24 sichere, dauernde Heilungen beobachtet: davon wurde $23 \mathrm{Mal}$ ausschliesslich das Thomas'sche Pessar angewandt; 1 Mal bei puerperalem Zustande ein Hodge'sches.

Die mittlere Tragzeit bis zur Heilung beträgt etwa 1 Jahr : die längste $2 \frac{1}{4}$ Jahre, die kürzeste in zwei puerperalen Fällen $21 / 2-3$ Monate.

Wird das Thomas'sche Pessar probeweise entfernt, so räth Fränkel, an dessen Stelle vor der Hand ein Wiegen-oder Hodge'sches Pessar einzulegen: es trete dabei eine allmälige Contraction des ausg eweiteten hinteren Scheidengewölbes ein. Erst wenn letztgenannte Stützapparate längere Zeit den Uterus in Anteversion halten, achtet er jedes Pessar für entbehrlich. Die theoretische Möglichkeit der Wiederherstellung der Muskelkraft des Retractor uteri während des Tragens eines Thomas schen Ringes wird 
praktisch bewiesen durch das Bleiben des Uterus in Normallage über einem kleinen Hodge-oder Wiegen-Pessar, die vor der Anwendung des Thomas'schen Pessars unwirksam waren.

Da jedes, auch das beste Pessar als ein nothwendiges Uebel anzusehen ist, so ist das Bestreben, auf operativem Wege eine Radikalheilung zu erzielen, leicht erklärlich. Indem Fränkel 118 Alexander-Adams'sche Operationen mit 3 Todesfällen, 14 völlig, anderen theilweise erfolglosen Resultaten ins Bereich seiner Betrachtung zieht, die Indicationsstellung kritisirt, kommt er zu dem Schlusse, dass diese Operation weitaus in der Mehrzahl der Fälle zu verwerfen sei. Als einzig berechtigte Indication lässt er zu: Complication von grossem Prolaps mit Retroflexio bezw. Cervixlaceration. Hier wird empfohlen: zunächst Anteversion des Uterus durch Anziehen der runden Bänder bezw. Cervixplastik in einer Session und in einer folgenden Kolpoperineorrbaphie, um späteres Einlegen eines Pessars und Dehnung der durch die Prólapsoperation oben verengten Scheide zu vermeiden.

Besprechung.

Küstner (Jena): Behufs Prüfung ihrer Wirksamkeit hat $\mathrm{K} \ddot{\text { ütner an }} 50$ verschiedenen Patientinnen mit reponiblen Retroflexionen nacheinander den Mayer'schen Ring, die Pessare von Hodge, Thomas und das 8-Pessar von Schultze eingelegt und dabei gefunden, dass der Mayer'sche Ring in 14\%, das Pessar von Hodge in $18 \%$, das von Schultze in $86 \%$, das von Thomas in $92 \%$ der Fälle wirksam war. In Bezug auf den Schultzeschen Ring glaubt Küstner, dass er wohl dasselbe, ja vielleicht noch mehr mit demselben erreicht haben würde als mit dem von Thomas, wenn er sich zu der nothwendigen Modellirung mehr Zeit gelassen haben würde.

Gegen die Adams'sche Operation spricht er sich auf Grund reicher Erfahrungen entschieden aus. Retroflexionen sind damit nicht zu heilen.

H ofmeier (Berlin) kann nicht bestimmte Zahlen anführen, ist aber allmälig ïber den Werth der Geradstellung bei der Heilung der Symptome der Retroflexion etwas zweifelhaft geworden. Bei den gewöhnlichen Hodge-Pessaren tritt allerdings häufig wieder Retroflexion ein; indessen sind trotzdem die Heilerfolge in Bezug auf die Symptome sehr gute. Er sieht aber den Erfolg nicht so sehr in der Geradstellung als in der Feststellung und der Verhinderung der Einklemmung des Uterus. Nur in der genauen Fest- 
stellung des Zusammenhanges zwischen den Retrodeviationen und den von $\mathrm{Hegar}$ sog. Lendenmarksymptomen können wir zu einer rationellen Therapie und einem Urtheil über ihren Heilerfolg gelangen. Daher ist auch jeder operative Eingriff mit grosser Vorsicht zu beurtheilen, ganz abgesehen von den zweifelhaften momentanen Erfolgen.

Sänger (Leipzig). Indem Sänger einen in der Leipziger geburtshülflichen Gesellschaft gehaltenen Vortrag, der dasselbe Thema behandelt, in Erinnerung bringt, betont er vor allem die prophylaktische Behandlung, welche er in dem Vortrage von Fränkel völlig vermisst. Da die postpuerperale Form der Retrodeviation weitaus die häufigste ist, so muss besonders im Wochenbette auf die Möglichkeit der Entstehung einer solchen Rücksicht genommen werden. Sänger hat es sich zum Grundsatze gemacht, alle Frauen, die er entbunden, in der zweiten bis dritten Woche zu untersuchen und, falls eine Rückwärtsverlagerung vorhanden, sie sofort durch künstliche Anteversion und Pessarbehandlung zu beseitigen. Derart frühzeitig entdeckte frisch postpuerperale Retrodeviationen gelangten nach seiner Erfahrung ausnahmslos binnen weniger Monate zur Heilung.

Czerny (Heidelberg) hat in zwei Fällen, ähnlich wie Olshausen, den retroflectirten, adhärenten Uterus an die vordere Bauchwand angenäht, nachdem er die entarteten Tuben und Ovarien entfernt hatte. Veranlassung zu diesem schweren Eingriffe gaben sehr schwere Retentio alvi et urinae, welche Symptome nach der Operation schwanden.

Veit hat gleichfalls gelegentlich von Operationen wegen Tubensäcken fixirte Retroflexionen bei der Laparatomie aufgerichtet und kann mit den Resultaten jetzt nach zum Theil $1 \frac{1}{2}$ jähriger Beobachtungszeit nicht gerade zufrieden sein, was die Lage anlangt, wenn auch die Symptome infolge der gleichzeitigen Castration nachliessen. Unter den Pessarien zieht er nicht zu kleine H odg e'sche Pessare, die er vorn, um das Herauskommen zu hindern, breit macht und die er für jeden Fall zurechtbiegt, um sie dann durch Celloidpessare zu ersetzen, allen anderen vor. Den ThomasOlshausen'schen Pessarien möchte er vorwerfen, dass sie vom Arzte nicht zurecht gebogen werden können. Die Zahl seiner dauernden Heilungen ist bei weitem grösser als die Zahlen Fränkel's. 
Frank hat, um Pessarien entbehrlich zu machen, folgende operative Verfahren angewandt, deren Resultate später veröffentlicht werden sollen. Er löste das Peritoneum an der vorderen Fläche des Uterus und an der hinteren Wand dor Blase los, legte dasselbe nach oben in eine Falte, befestigte es so durch subcutane Catgutligaturen und brachte dadurch die vordere wunde Uteruswand mit der hinteren des Peritoneums entblössten Blasenwand in Berührung. In anderen Fällen präparirte er einen Lappen aus der vorderen Abdominalwand mit der Basis nach oben und heilte seine Spitze an die durch Loslösen des Peritoneums wund gemachte vordere Uteruswand an.

Schwarz (Halle): In jenen Fällen, wo infolge grosser Schlaffheit des Uterus sowohl als seines Bandapparates Vaginalpessarien nicht im Stande sind, den Uterus in seiner richtigen Lage zu erhalten, fixirt $\mathrm{Schwarz}$ den Uterus durch einen gewöhnlichen Mayer'schen Ring oder ein Wiegenpessar und bekämpft gleichzeitig den mangelhaften Tonus des Uterus durch Injectionen von Liquor Ferri und adstringirende Flüssigkeiten. Die Radikalheilung erfolgt bei dieser Therapie viel rascher und sicherer als bei blosser orthopädischer und sind dabei Monstrepessarien oder complicirte Formen solcher ganz entbehrlich.

Ilgner (Elbing) sucht den Grund der ungünstigen Conceptionsbedingungen bei reponirten Retroflexionen in der dabei sehr ungünstigen Stellung des Orificium uteri externum. Deshalb empfiehlt er nach Schwinden der Beschwerden eine zeitweise Entfernung des Pessars. Trete dann Conception ein, so könne man im darauffolgenden Puerperium am ehesten bei zweckmässiger Behandlung auf endgültige Heilung hoffen.

Martin (Berlin) ist der Ansicht, dass man bei chronischen Retrodeviationen am besten nur so lange und nur dann eine Pessarbehandlung anwenden soll, als dieselbe Symptome macht. Eine weitere Indication zur Geradstellung könne gegeben sein durch Sterilität. Frische Formen heilen oft von selbst. Martin zieht das Hodge'sche Pessar den anderen vor.

Fränkel spricht sich nochmals zu Gunsten präformirter Pessarien aus, die namentlich für den praktischen Arzt viel leichter zu handhaben als andere Formen. Extreme in Bezug auf Grösse der Ringe sind selbstredend zu vermeiden.

Anbetreffend die Existenz ron Lendenmarksymptomen bei Retrodeviationen giebt er deren Zustandekommen durch gleich- 
zeitige Erkrankungen der Adnexa selbstredend zu, betont aber andererseits, dass bestehende oft rasch verschwinden nach Beseitigung der Lageanomalie.

Den Werth der von Schwarz empfohlenen tonisirenden Behandlung anerkennt er vollkommen, ebenso die Prophylaxe im Wochenbette. Dagegen ist er der Ansicht, dass eine bestehende Retrodeviation im Wochenbette fast nie heile. Nicht die Retroflexio, sondern deren Folgezustände sind Ursache der bei dieser Lageanomalie so häufig beobachteten Sterilität.

5) Kehrer (Heidelberg): Ueber Inversio uteri. Redner unterscheidet zwei Formen von Inversio uteri.

1) Es ist der Fundus uteri an gewöhnlicher Stelle stehen geblieben oder sogar höher gestiegen, und ist nur die Corpusmucosa allein oder zusammen mit den innersten Muskelschichten eingestülpt durch ein Myom.

2) Es ist, indem die ganze Dicke der Uteruswand eingestülpt ist, ein richtiger Peritonealtrichter vorhanden.

In ersterem Falle besteht die Behandlung in Enucleation des Myom mit nachfolgender Vernähung der resecirten Geschwulstdecke.

Im zweiten Falle ist die Amputation in Betracht zu ziehen.

Vortragender weist ein Präparat vor, herrührend von einer Patientin, die er vor kurzer Zeit operirt hat. Ein zweifaustgrosser Tumor, bestehend aus dem mit Myomen besetzten invertirten Uterus, füllte die Vagina aus. Es w urde durch Herabziehen des Tumor ein Prolapsus bewirkt, der Stiel etwas unterhalb des Orificium internum amputirt und Bauchfelltrichter und Muskulatur getrennt durch Nähte vereinigt. Der Stumpf wurde mittels der zusammengeknüpften tiefen Nähte weit unten in der Scheide gehalten. Nach Entfernung derselben trat in der Folge eine spontane Reinversion ein. Vollkommene Heilung.

Besprechung.

Schroeder verfährt in den Fällen, wo sich durch Myome eine Inversion gebildet hat, so, dass er den Uterus partiell oder sogar vollständig weiter invertirt. Auf dem so dem Auge zugänglich gemachten Tumor wird dann die Schleimhaut da, wo sie auf den Tumor übergeht, incidirt und das Myom enucleirt. Der Uterus geht dann von selbst zurück oder lässt sich leicht reponiren. Auf diese Weise wird die Amputation überflüssig. 
322 Verhandlungen der gynäkologischen Section der 59. Versammlung

Kehrer rechtfertigt die Amputation in seinem Falle damit, dass die Muscularis, welche die Myome einhüllte, derart hypertrophisch gewesen sei, dass an eine Enucleation mit nachfolgender Reposition nicht gedacht werden konnte.

Fritsch (Breslau) hat in einem Falle von Amputation bei Inversion den invertirten Uterus vorher mit der Portio vernäht und dann abgetragen. Dadurch wurde ein Hineinragen der blutenden Amputationsfläche in die Bauchhöhle vermieden. Nach Umstechung der blutenden Gefässe wurde mit Jodoformgaze tamponirt. Patientin genas.

6) Frank (Cöln): Ueber Lappentransplantation bei Stenosenoperationen.

Bei Stenosen, welche einen grossen Theil des Cervicalkanales betreffen, und bei solchen des inneren Muttermundes empfiehlt Frank eine Reihe von Operationen, bei welchen ein Lappen aus der äusseren Fläche der Portio in den Cervicalkanal eingenäht wird. Auf diese Weise sollen Stenosen dauernd beseitigt und namentlich die lästigen Dilatationen nach anderen Methoden vermieden werden. Nach seinen Methoden hat Frank bereits zehn Fälle mit gutem Erfolge operirt.

Besprechung.

Fritsch bezeichnet die Frank'schen Vorschläge für überflüssige Künsteleien und bezweifelt sehr, dass bei diesen Operationen der innere Muttermund getroffen wird.

Frank behauptet in Bezug auf letzteren Punkt das Gegentheil. Auch soll die Operation nicht blos wegen Sterilität in Anwendung kommen, sondern ihr Hauptzweck bestehe darin, die Patientinnen von ihren Beschwerden zu heilen und, wie im Vortrage erwähnt, die lästige Nachbehandlung überflüssig zu machen.

7) Bockelmann (Berlin): Ueber Antisepsis in der Geburtshülfe.

Der Vortragende giebt eine kurze Darstellung der antiseptischen Grundsätze, welche an der Berliner Universitätsfrauenklinik in Geltung sind. Das Hauptgewicht wird immer mehr auf die Desinfection des Geburtshelfers gelegt, während die Desinfection der Kreissenden auf ein geringes Maass beschränkt werden kann. Die Desinfection der Hände wurde mit einer Su- 
blimatlösung von $1: 1000$, diejenige der Instrumente mit 3- bis 5 procentiger Carbolsäure vorgenommen. Nur zu Beginn der Geburt fand eine Scheidenausspülung mit einer Sublimatlösung statt; ebenso vor der Vornahme geburtshülflicher Operationen. Unmittelbar nach der Geburt wurden Scheiden- und Uterusausspülungen nur auf stricte Indication gemacht. Im weiteren Verlaufe des Wochenbettes war die Behandlung eine rein exspectative, auch hier wurden nur bei strenger Indication desinficirende Ausspülungen des Genitaltractus vorgenommen. Sublimatintoxicationen sind, seit schwächere Lösungen in Anwendung kamen, überhaupt nicht mehr beobachtet.

Besprechung.

Meer mann (Mannheim) begrüsst die in letzten Jahren immer mehr zu Tage tretende Abneigung gegen die früher übliche Polypragmasie. In Bezug auf die Hebammen möchte er ebenso jede Antisepsis an der Kreissenden vermieden, gründliche Desinfection der eigenen Person empfohlen haben.

Schatz (Rostock) hält die gesunde Kreissende nicht wie Bockelmann für aseptisch und erachtet deshalb gründliche Desinfection der Vagina vor der Geburt für sehr zweckmässig.

Korn (Dresden) pflichtet der Ansicht von Schatz bei. Er empfiehlt im Ferneren jeder intrauterinen Operation eine Uterusausspülung folgen zu lassen. Dagegen sollen derartige Manipulationen im Wochenbette möglichst eingeschränkt und nur die Vagina, der gewöhnliche Ort der Zersetzung, ausgespült werden.

\section{Sitzungstag: Dienstag, den 21. September.}

A. Demonstrationen in der geburtshülflich-gynäkologischen Universitätsklinik der Charité.

Hennig (Leipzig) weist Nachbildungen des Altenburgischen Mädchens mit Wirbelverlängerung an zwei Wachsabgüssen und zwei Gypsformen vor. Dem Vortragenden ist es gelungen 22 hierher gehörige Beispiele aus der Literatur zusammenzustellen; darunter fand sich nur eines, wo die Schwanzbildung als Familienanlage bestand.

Die Untersuchungen, welche das Altenburgisch e Mädchen betrafen, führten zu dem Resultate, dass demselben das permanente Schwanzbein fehle. Rauber, welcher die mikroskopi- 
sche Untersuchung des vorliegenden Präparates vornahm, nimmt an, dass der unterste Wirbel des nur aus vier Stücken bestehenden Kreuzbeines an diesem Präparate zugleich als Rückbleibsel eines sacralen Steissbeinstiuckes aufzufassen sei. Den Schwanz fand er durch zwei gelenkig unter einander verbundene Längsknochen. gestützt, welche grösstentheils knorpelig sind. H ennig glaubt, dass es sich hier um Verschmelzung ursprïnglich distaler Steisswirbel in zwei bleibende handelt. Der wichtigste Befund ist die Darstellung eines centralen Ganglion des Grenzstranges in der unteren Schleife des sympathischen Nerven und besonders eigener Muskeln des Schwanzes, namentlich ein Hebemuskel durch Rauber. Der Mastdarm mündet winzig in den Scheidenvorhof. Die unteren Gliedmaassen vereinigen Spalten des robbenartigen Fusses, rechts in anderem Sinne als links, mit Verschmelzung mittels feiner Fäden, die als amniotische nicht erwiesen sind. Ausfall von Zehen, Verdoppelung der grossen Zehe; die Zehen sind kurz, dünn mit Ausnahme der äusseren, welche verlängert sind und zugleich Nagelmangel zeigen. Der linke Unterschenkel ist in trauterin nach hinten luxirt. Wichtig ist in dem Falle der Mangel an Fruchtwasser. Auf die dadurch geschaffene Raumbeschränkung sind wohl die Abnormitäten der unteren Extremitäten zurückzuführen, während die bestehenden Muskelfehler sich daraus nicht erklären lassen.

Derselbe Redner demonstrirt einen Fall von hochgradiger angeborener Rhachitis. Der nach der Geburt rasch gestorbene Knabe zeigte überlangen Kopf und Rumpf: die Wirbelsäule im Brusttheile mehrfach zickzackartig geknickt. Filum terminale doppelt. Darmbeinschaufeln nach vorn unten ohrförmig herabgebogen; es mangeln die vorderen Stacheln; hinten sind die sehr kleinen schmalen' Schaufeln hinter die Facies auriculares der Kreuzbeinflügel geschoben. Die Conjugata vera des stark geneigten Beckens beträgt 6 statt wie normaliter $24 \mathrm{~mm}$; der Querdurchmesser des Einganges $38 \mathrm{~mm}$.. Die Darmbeinplatte ist $40 \mathrm{~mm}$ lang, 28 breit, 8 dick. Schwanzbein knorpelig, vorn $10 \mathrm{~mm}$, hinten ganz frei. Dasselbe ist $30 \mathrm{~mm}$ lang, 15 breit, 1 dick (statt 18:10:7), und enthält 51/2 Wirbel, dagegen keine Muskeln.

Fürst (Leipzig): 1) Demonstration eines Glühlichthalters zu gynäkologisch-diagnostischen Zwecken. 
Derselbe ist sehr leicht an einem Röhrenspeculum anzubringen und befindet sich die Lichtquelle zwischen Auge und Portio. Ein Reflector verhindert die Blendung des Auges; die Hände des Arztes bleiben frei und ist durch den Apparat das Gesichtsfeld weder beeinträchtigt noch werden kleinere Manipulationen an der Portio durch denselben wesentlich beschränkt.

2) Demonstrationen von Modellen der Vulva und der Portio vaginalis (siehe Deutsche medicinische Wochenschrift 1885, Nr. 46).

Gottschalk (Berlin) legt der Gesellschaft mehrere von Landau auf operativem Wege gewonnene Präparate vor:

a) ein Bauchdeckensarcom, das sich bei einer IIIpara während der letzten Schwangerschaft entwickelt hatte. Dasselbe ging aus von einer Inscriptio tendinea des linken Musculus rectus, der lang ausgezogen über den Tumor wegzog. Er war mit dem Muskel sowohl als dem Peritoneum fest verwachsen. Die Exstirpation gelang ohne Eröffnung der Bauchhöhle;

b) ein innerhalb des Mesenterium des Dünndarmes entwickeltes Kystom. Es lag der Aorta direct auf und dachte man infolge der dadurch deutlich hörbaren Herztöne an Extrauteringravidität. Die Cyste war sehr beweglich, enthielt völlig klaren, nicht cystösen Inhalt;

c) Fall von Ovarialschwangerschaft, herstammend von einer 26 jährigen, seit drei Jahren steril verheiratheten Frau. Der als Ovarialtumor diagnosticirte Tumor erwies sich als Graaf'scher Follikel. In ihm lag der kaum 0,7 cm lange Embryo auf dem Dottersacke in dem Amnion. Die $1 \mathrm{~cm}$ lange Nabelschnur ging in die Kystomwand hinein. Tube und anderes Ovarium gesund;

d) primäres Sarcom der rechten Tube. Bei der Operation bestanden bereits Metastasen in der Form kleiner derber Knoten auf dem Peritoneum. Der Tumor war über wallnussgross, sass im abdominalen Theile der Tube, erwies sich bei der mikroskopischen Untersuchung als Spindelzellensarcom;

e) Präparat von Tubengravidität aus dem zweiten Monat; die Tube war geborsten;

f) Präparat von Eileiterschwangerschaft aus dem zweiten Monate mit sehr starker Verdünnung des Fruchtsackes; 
326 Verhandlungen der gyuäkologischen Section der 59. Versamminng

g) gravider Uterus, wegen Cancroid der Portio exstirpirt.

Lan da u (Berlin) empfiehlt der Versammlung seine be i Häng e bauch und nach Laparatomien in Anwendung kommende Leibbinde. Dieselbe besteht aus nur durch zwei Federn beiderseits gebogener Pelotte, und hat die Tendenz, die Kraft des auseinandergewichenen und atrophischen Rectus abdominalis zu ersetzen.

Die Leibbinde wirkt also wesentlich von der Seite her und nicht wie bei den gewöhnlichen Bịden von vorn.

Wyder (Berlin) legt unter Vorweisung einiger Zeichnungen eine Modification der Demonstration makro-und mikroskopischer Bilder vor. Die Zeichnungen, auf starkes Pausepapier übertragen, werden zwischen zwei in einem Rahmen befindliche Glasplatten gelegt und bei durchfallendem Lichte betrachtet. Der Unterschied zwischen dieser Art der Zeichnungen und der auf gewöhnlichem Papier ist ungefähr derselbe wie der zwischen einem Stereoskopbild auf Glas und Papier. In nächster Zeit soll im Verlage von Fischer's medicinischer Buchhandlung in Berlin ein Atlas für Kliniker, vorzugsweise aus mikroskopischen Bildern bestehend, nach dieser Modification herausgegeben werden.

Langner (Berlin): 1) Fall von kleinzelligem Rundzellensarcom der Uterusschleimhaut. Das Präparat war durch supravaginale Amputation des Uterus gewonnen und ist bis jetzt Patientin frei von Recidiv geblieben. Die Operation wurde vorgenommen wegen der starken, jeder anderen Therapie trotzenden Blutungen.

2) Fall von Nierencarcinom. Wegen Mangel aller für eine Nierenerkrankung sprechender Symptome war keine bestimmte Diagnose gestellt worden. Laparatomie. Es stelite sich heraus, dass ein Nierencarcinom vorlag. Der Tumor wurde so viel wie möglich ausgeschält, das Bett vernäht. Tod am elften Tage nach vorausgegangenex Anurie. Bei der Section fand sich ein Abscess an der Stelle des Tumor, keine Peritonitis. Der linke Ureter war normal, die zugehörige Niere fettig degenerirt, jedoch nicht in einem Grade, welcher die Anurie erklären konnte.

3) Fall von Hydrosalpinx. Früher Salpingotomia sinistra mit drei Monate langem Wohlbefinden (Erkrankung auf go- 
norrhoischer Basis beruhend). Später zweite Laparatomie und Entfernung einer rechtsseitigen Hydrosalpinx. Bei der ersten Operation war die rechte Tube noch völlig gesund gewesen.

Orthmann (Berlin): Demonstration eines Falles von vereitertem Myom des Uterus mit Perforation. Nachdem bei der 42 jährigen Patientin die Diagnose auf vereiterndes Uterusmyom gestellt worden, wurde bei starkem Fieber die supravaginale Amputation vorgenommen. Der Uterus erwies sich bei der Operation fest mit dem Kreuzbeine verwachsen und drang bei dem Versuche, ihn zu lösen, übelriechender Eiter aus dem ersteren, zum Theil in die Bauchhöhle. Das Myom hatte an der Verwachsungsstelle die Wand durchbrochen. Am vierten Tage erfolgte der Tod an septischer Peritonitis. Bei der Section zeigten sich folgende Verhältnisse. Die ganze Uterushöhle wird von einem in jauchigem Zerfalle begriffenen, 6 bis $8 \mathrm{~cm}$ im Durchmesser messenden Myom eingenommen. Der Zwischenraum zwischen Uteruswand und Tumor wird von stinkendem Eiter ausgefuillt; die Uteruswand ist mit eitrigem Belag versehen; der Tumor ist im Fundus und links fest mit der Uteruswand verwachsen; auf der Höhe des Fundus befindet sich eime 2 bis $3 \mathrm{~cm}$ fassende Perforationsöffnung, welche von der Tumormasse zum Theil ausgefüllt wird; in der vorderen Uteruswand befinden sich noch zwei intraparietale und ein subseröses Myom von 2 bis $3 \mathrm{~cm}$ Durchmesser.

Baumgärtner (Baden-Baden) weist ein Bidet mit Apparat für Scheidenausspülung versehen vor. Das Bidet, mit Rücklehne versehen, ist so eingerichtet, dass sich die Patientin, bequem und richtig gelagert, längere Zeit ohne Ermüdung Scheidenausspülungen machen kann. Das Vaginalrohr ist leicht einstellbar. - Der Apparat, welcher auch mit einem Badeofen in Verbindung gesetzt werden kann, ist in der städtischen Frauen. klinik zu Baden-Baden mit gutem Erfolge in Gebrauch.

\section{B. Vorträge in der Universitätsaula.}

8) Gusserow (Berlin): Exstirpation von Tubensäcken.

Gusserow berichtet über seine Erfahrungen über Pyosalpinx, gewonnen aus 14 Fällen derartiger Erkrankung, die zur Operation kamen. In sämmtlichen Fällen waren perimetritische $\mathrm{Zu}-$ stände vorhanden. Diese Perimetritis ist ätiologisch jedenfalls als 
328 Verhandlungen der gynäkologischen Section der 59. Versammlung

das Wesentliche zu betrachten und in den meisten Fällen entstanden durch gonorrhoische Infection, welche zu eitrigem Tubenkatarrh und dann zu Entzündung der Serosa der Uterusadnexa geführt hat. Die Folge der adhäsiven Entzündung ist Verschluss der Tube und Ansammlung des Eiters (Pyosalpinx). Ebenso wird das Krankheitsbild wesentlich durch den Grad der Perimetritis bedingt, je intensiver diese, um so heftiger die Schmerzempfindungen, die Dysmenorrhoe und gewöhnlich auch die kolikähnlichen Anfälle, die charakteristisch für diese Zustände sind. Ein nicht unwesentliches Symptom bilden noch die profusen Menstrualblutungen, welche wohl durch die Circulationsstörungen zu erklären sind, bedingt durch die Entwickelung der Tubensäcke im Ligamentum latum. Die Bedeutung der Krankheit liegt ebenfalls in den perimetritischen Zuständen. Die Gefahr der Berstung und dadurch bedingten Peritonitis ist nach den Erfahrungen des Vortragenden sehr gering. Wiederholt war es bei den Operationen nicht zu vermeiden, dass der Inhalt der Tubensäcke in die Bauchhöhle kam, ohne dass dieser Umstand irgendwie die vollständige Heilung beeinflusst hätte.

Es wird demgemäss die Indication zur operativen Entfernung dieser Tubensäcke wesentlich von der Intensität der perimetritischen Beschwerden abhängen, nur dürfte dabei zu erwägen sein, dass nicht immer mit Entfernung der Tubensäcke diese Beschwerden vollständig verschwinden. Wichtig ist es dabei, die Ovarien mitzuentfernen.

Die 14 vom Vortragenden operirten Fälle genasen sämmtlich. (Der Vortrag wird anderweitig ausführlich veröffentlicht werden.)

9) Martin (Berlin): Ueber Tubenerkrankungen.

Redner verfügt über 287 Fälle von Tubenerkrankungen, aus denen hervorgeht, dass Entzündungsprocesse an den Tuben zwar recht häufig sind, dass man aber über die Hälfte der Fälle auffassen muss als das Product einer fortschreitenden katarrhalischen Affection der Uterusschleimhaut. Die übrigen Fälle gehören zur einen Hälfte der puerperalen Erkrankung an, zur anderen der gonorrhoischen bezw. tuberkulösen in einem Verhältnisse wie 6:1.

In Bezug auf die Localisation handelt es sich

in 91 Fällen um doppelseitige,

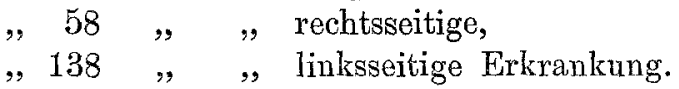


Man kann deutlich zwei Formen von Salpingitis unterscheiden:

1) Endosalpingitis interstitialis,

2)

follicularis, wobei die Tubenwand von cystischen Räumen durchsetzt erscheint.

Die weiteren Veränderungen spielen sich ab:

1) in der Wand der Tube: der Muskulatur und dem peritonealen Ueberzuge;

2) im Lumen als Folge der Stenosirung und Atresie;

3) als Resultat der Entwickelung von Mikroorganismen (gonorrhoischer, puerperaler, tuberkulöser, actinomycotischer Natur).

Heilung kann eintreten und sogar nachher noch Gravidität erfolgen. Pathognomonische Zeichen sind nicht vorhanden; die hauptsächlichsten sind Schmerzen, Blutungen, Sterilität. Die Therapie muss zunächst eine antiphlogistische sein; erst wenn dieselbe erfolglos bleibt, darf die operative in Frage kommen. Unter seinen Fällen war Martin 64 Mal in der Lage, die Salpingotomie vornehmen zu müissen.

10) Meinert (Dresden): Klinisches über die Exstirpation von Tuben und Tuboovarialsäcken.

Redner hat 15 Salpingotomien ausgeführt, dadurch 14 Patientinnen zur Genesung gebracht; nur 1 starb an Tetanus. $7 \mathrm{Mal}$ handelte es sich um Pyo-, 7 Mal um Hydrosalpinx, 1 Mal um einen rupturirten Fruchtsack bei Eileiterschwangerschaft.

Die meisten Tubarsäcke führt Meinert auf gonorrhoischen Ursprung zurück; jedoch gelang es in drei Fällen, die sich im Anschlusse an eine starke Scheidengonorrhoe entwickelten, Prof. Neelson nicht, Gonococcen in den Präparaten nachzuweisen. Fälle von tuberculöser Salpingitis sah Vortragender zwei Mal bei gleichzeitiger peritonealer Tuberkulose. Das Tubensecret braucht selbst bei Pyosalpinx nicht giftig zu sein. Bei einer spontan geplatzten Pyosalpinx mit nashfolgender Peritonitis laparatomirte er mit Erfolg. Im Ferneren berichtet Redner über einen Fall, wo die fibrinösen. Ablagerungen aus dem ausgetretenen Tubensecret eine Abschnürung des Processus vermiformis und Perforativperitonitis erzeugten. Die Symptome der Tubenerkrankungen sind veränderlich und ungleich. Sehr häufig ist das Nervensystem angegriffen. Die Grösse der Tumoren steht zur Heftigkeit der Symptome in keinem 
330 Verhandlungen der gynäkologischen Section der 59. Versammlung

Verhältnisse. Je stürmischer die Initialsymptome der gonorrhoischen Salpingitis, desto schwerer gewönnlich der Verlauf. Frische höchstgradige gonorrhoische Pelviperitonitis wurde durch Exstirpation von Tuben und Ovarien geheilt. Tubenhydrops selbst von beträchtlicher Ausdehnung kann sich binnen wenig Wochen entwickeln. Ausfliessende Tubensäcke wurden vom Redner drei Mal beobachtet, zwei Mal operirt.

11) Czempin: Ueber die Beziehungen der Uterusschleimhaut $z u$ den Erkrankungen der Adnexa des Uterus.

Unter den Erkrankungen der Uterusadnexa, welche eine Erkrankung der Uterinschleimhaut setzen können, spielen eine Rolle:

1) Recidivirende acute oder subacute Erkrankung der Ovarien, der Tuben oder beider Organe zugleich;

2) exsudative Parametritis;

3) pelvi-peritonitische Reizzustände von Narben der Ligamenta nach Ovario- oder Salpingotomien;

4) gewisse Tumorenbildungen der Adnexa, besonders Pyosalpinx und Ovarialsarcome.

Dic genannten Zustände können alle Veränderungen der Uterusmucosa im Gefolge haben, welche sich meistẻns äussern in atypischen heftigen Blutungen, deren Charakter jedoch meist bei den verschiedenen Formen der Adnexaerkrankungen ein verschiedener ist. Bei Pyosalpinx, Ovarialsarcom und einigen Formen von Tubei - und Ovarialerkrankungen bestand Endometritis hyperplastica glandulärer und interstitieller Natur. In anderen Fällen zeigte die Schleimhaut geringe oder gar keine Veränderungen und waren wohl hier die Blutungen wesentlich bedingt durch arterielle Congestion, verursacht und unterhalten durch die Erkrankung der Anhänge.

Besprechung der Vorträge von Gusserow, Martin, Meinert und Crempin.

Hegar weist zunächst auf die Häufigkeit der Tubenerkrankungen hin und betont, dass gewöhnlich eine Affection der $\mathrm{Mu}$ cosa des Sexualschlauches das Primäre sei. Die Einwirkung der Adnexa und des Ligamentum latum auf die Mucosa, speciell des Uterus, wie sie von $\mathrm{Czempin}$ beleuchtet, sei eine altbekannte Thatsache. Im Speciellen habe Hegar immer darauf hingewiesen, 
wie durch Exsudatknoten, Schwielen, entzündliche Abkapselungen des Sclinürstückes nach Castration atypische oder typische Blutungen auftreten können, welche das Resultat der Operation zu trüben im Stande sind.

In Bezug auf die Symptomatologie weist Hegar darauf hin, dass die Tumoren oft gar keine oder nur geringe Beschwerden machen, dass häufig Sterilität die Ursache sei, warum der Arzt berathen werde. Auch er hält die dabei so häufig beobachtete Pelviperitonitis für denjenigen Zustand, der den ganzen Symptomencomplex beherrsche. Deshalb sei auch Vorsicht geboten in Bezug auf die Indicationsstellung. Schreite man zur Operation, so sei nicht allein die Exstirpation der Tubarsäcke, sondern namentlich der Ovarien die Hauptsache: also nicht die Salpingotomie, sondern die Castration, da eben, wie bereits angedeutet, gewöhnlich nicht die Tubengeschwulst, sondern die immer wiederkehrende Pelviperitonitis Beschwerden veranlasse. Aus dem Grunde sei aber auch das Endresultat der Salpingotomie, namentlich bei Pyosalpinx, durchaus nicht immer ein glänzendes und müsse man befriedigt sein, wenn vor der Hand nur ein Nachlass der bestehenden Beschwerden der Operation folge.

Eine Ruptur der Tubarsäcke und den Austritt des Inhaltes in die Bauchhöhle hält er nicht stets für bedeutungslos, dagegen spreche die nach einem derartigen Ereigniss beobachtete tödtlich verlaufende Peritonitis; im Ferneren die Jauchherde, welche man gelegentlich in der Umgebung selbst nichtgeplatzter Tubensäcke beobachten könne.

Kaltenbach (Giessen) macht aufmerksam auf die verschiedenen Schwierigkeiten, welche Erkrankungen der Tuben in Bezug auf Aetiologie, Diagnose, Verlauf bieten und die daher eine bündige Indicationsstellung zur Zeit nicht gestatten.

In Bezug auf die Diagnose weist er im Speciellen auf die Aebnlichkeit hin, welche schnell wachsende Tubentumoren mit Entfaltung der Ligamenta lata mit anderweitigen intraligamentären Geschwülsten und Ergüssen haben können und erwähnt zur Illustration eines Falles, wo zwei Tubensäcke von Eigrösse innerhalb Jahresfrist bis zu Kindskopfgrösse wuchsen, die während der Operation platzten und Tod an heftigster Sepsis bedingten.

Im Weiteren erwähnt Kaltenbach die verschiedenen Arten der Ruptur der Tubensäcke: im Anschluss an einen geringfügigen intrauterinen Eingriff; spontan durch zeitweises Nachgeben der 


\section{Verhandlungen der gynäkologischen Section der 59. Versammlung}

lose verklebten Abdominalöffnung secretgefüllter Tubensäcke. Ferner hat Kaltenbach nach gonorrhoischer Infection eine grössere mit starker Peritonitis pelvica complicirte Pyosalpinx in der freien bezw. in der mangelhaft durch Verklebung geschützten Bauchhöhle platzen sehen, wodurch ein grosses intraperitoneales Exsudat veranlasst wurde. Er kann wie Hegar den Inhalt der eitrigen Tubensäcke nicht für so unschuldig halten, wie es von einer grossen Anzahl ron Gynäkologen geschieht.

In Bezug auf die Therapie ist Kaltenbach der Ansicht, dass viele Tubarsäcke gar nicht Gegenstand operativer Behandlung sind und dass da, wo die Entfernung sehr wünschenswerth wäre, dieselbe recht gefährlich oder technisch undurchführbar sein kann.

Schroeder warnt ebenfalls davor in allen Fällen, wo eine Tubenerkrankung diagnosticirt worden, zur Laparatomie zu schreiten. Es gäbe Fälle, wo bei starken Beschwerden geringe Veränderungen der Tuben und Ovarien gefunden werden und vielleicht eine conservative Behandlung am Platze gewesen wäre. Andererseits müsse daran erinnert werden, dass selbst- eine Pyosalpinx durch Eindickung des Inhaltes unschädlich gemacht werden könne.

Olshausen ist es aufgefallen, wie oft Tubengeschwïlste sich auffallend wenig nach abwärts in die Ligamenta lata hinein entwickeln und den Uterus viel weniger in die Höhe ziehen als manche subserös entwickelten Ovarialkystome.

In Bezug auf die Genese macht ex darauf aufmerksam, dass manche Pyosalpinx da auf tuberkulöser Basis beruh乞, wo man an diese Möglichkeit gar nicht gedacht hat. Deshalb sei es wohl am Platze, bei Pyosalpinx nur da auf gonorrhoischen Ursprung zurück zu gehen, wo die Anamnese sichere Anhaltspunkte ergebe und die Untersuchung auf Tuberkulose völlig negatives Resultat zur Folge habe.

Löhlein sieht in der Häufigkeit der Tuberkeleruption auf der Tubenschleimhaut bei völlig gesunder Uterusmucosa einen Beweis für die Richtigkeit der Auffassung Hegar's, dass die Entzündungserreger besonders aus den Lymphgefässen der Ligamenta lata in die Tube einwandern. Vielleicht liegt in dieser Art auch die Erklärung für das härfigere Befallensein der linken Tube, da für die Parametritis diese numerische Bevorzugung ja bekannt ist.

Martin anerkennt die Salpingotomie nur als letzte Zuflucht und will Tubenerkrankungen im Allgemeinen symptomatisch be- 
handelt wissen. Fälle von Conception nach Salpingitis sind die besten Anhaltspunkte für eine conservative Behandlung.

12) J. Schramm (Dresden): Ueber Castration bei Epilepsie.

Eine Zusammenstellung der bei Epilepsie und Hysteroepilepsie ausgeführten Castrationen zeigt, dass die Resultate der Operation bei ersterer weniger günstig sind als bei anderen Krampfformen. Immerhin kann sich die Castration gesunder Ovarien nach Redner als Heilmittel gegen epileptische Zustände erweisen, wenn dieselben mit dem Menstruationsprocess in Zusammenhang stehen. Zwei schwere hierher gehörige Fälle hat er vor 1 resp. $1^{1} /_{2}$ Jahren castrirt und sind bis jetzt weitere epileptische Anfälle nicht mehr eingetreten. (Der Vortrag wird ausführlich anderweitig veröffentlicht werden.)

13) Schroeder: Ueber die Castration bei Neurosen.

Hegar trennt sehr richtig die Herausnahme kranker Eierstöcke von der Exstirpation derselben, um die Menopause herbeizuführen.

Die Indicationen zur Operation im ersten Falle sind klar und bieten grundsätzlich gar keine Schwierigkeiten, da sie allgemein chirurgische sind. Man entfernt die Ovarien, weil sie entartet sind (grosse und kleine Tumoren), aber auch in anderen pathologischen Veränderungen (chronische Oophoritis). Ist nur ein Ovarium krank, so ist nur die einseitige Exstirpation angezeigt; ja unter Umständen nur die theilweise.

Ganz anders steht es mit der Exstirpation der Ovarien, um die Menopause vorzeitig herbeizuführen. Hier müssen natürlich stets beide Ovarien vollständig entfernt werden. Der Zweck wird auch erreicht, wenn die Ovarien gesund sind. Trotzdem kommt $\mathrm{Hegar}$ auf indirectem Wege dahin, die Castration nur bei pathologisch-anatomischen Veränderungen der Eierstöcke oder anderer Theile des Genitalkanales zuzulassen. Er kommt dahin, weil er die Exstirpation nur in ihrer Rüickwirkung auf die Genitalien als berechtigt anerkennt, oder wenn Neurosen durch pathologische Veränderungen in den Ovarien bedingt sind.

Dies kann ich nicht als berechtigt anerkennen. Zunächst ist es ein grosser Unterschied, ob man castrirt, weil die Ovarien krank sind oder weil der Uterus einen Tumor enthält. Der erste Fall gehört nicht zur Castration im engeren Sinne; im zweiten 
334 Verhandlungen der gynälzologischen Section der 59. Versammlung

Falle entfernt man ein gesundes Organ, um eine Rïckwirkung auf ein anderes krankes Organ auszuüben.

Ganz ebenso kann man auch bei allgemeinen Neurosen gesunde Eierstöcke entfernen, um eine heilsame Rückwirkung auf den Gesammtorganismus auszuïben.

Ob und inwieweit Neurosen von pathologischen Veränderungen in den Eierstöcken abhängig sind, wissen wir noch nicht. Hegar selbst hebt hervor, dass man bei sehr bedeutenden pathologischen Veränderungen dieselben nicht findet. Aber auch geringe Veränderungen machen dieselben oft gar nicht, in anderen Fällen sind nur Rückwirkungen da, wie man sie auch bei anderen die Gesundheit und den Lebensgenuss beeinträchtigenden Leiden findet.

Schroeder behauptet, dass schwere Neurosen nach der natürlichen oder künstlichen Menopause schwinden können, ohne dass deutlich pathologische Veränderungen in den Ovarien vorhanden sind.

Die Hegar'sche Forderung der pathologischen Veränderungen hat aber auch eine grosse Schwierigkeit in unserer Unkenntniss über das, was am Ovarium noch als innerhalb normaler Verhältnisse liegend anzusehen ist. Uns fehlen noch alle Vorarbeiten, um zu beurtheilen, wann kleine Cysten in den Eierstöcken, wann eine derbere Beschaffenheit des Stroma als pathologisch zu betrachten ist.

Ebenso wie bei Uterusmyomen nur durch die Erfahrung festgestellt werden konnte, dass die Entfernung der gesunden Ovarien heilend auf die Myome wirkt, ebenso kann die Frage, ob die Entfernung gesunder Ovarien bei Neurosen Vortheil bringt, nur durch Erfahrungen entschieden werden.

Deswegen will ich einige Krankengeschichten kurz als Belege mittheilen.

Ich habe zwölf Mal aus dieser Indication operirt, kann jedoch noch nicht alle verwerthen, da sich ein sicheres Resultat erst nach Jahren feststellen lässt. Drei Fälle mit sehr günstigem Resultate sind aber $8 \%, 7$ und 5 Jahre alt. Zwei von diesen haben geheirathet.

Aus diesen Fällen geht hervor, dass bei Ovarien, die vom. normalen Verhalten nicht abweichen, schwere Neurosen durch die Castration Heilung finden können. Unsere Aufgabe wird es sein, die Fälle genauer zu bestimmen, in denen dies erwartet werden 
kann. Nach meiner Erfahrung ist dies besonders dann der Fall, wenn in der Aetiologie geschlechtliche Verhältnisse eine hervorragende Rolle spielen.

Besprechung der Vorträge von Schramm und Schroeder.

Hegar weist in erster Linie zwei ihm untergelegte Ansichten zurück. Er hat die Castration gesunder Ovarien wegen Neurosen nicht verworfen, sondern nur verlangt, dass ein causaler Zusammenhang einer Neurose mit einer pathologischen Veränderung im Bereiche des Sexualapparates nachgewiesen werde. Ebenso ist er auch nicht absolut gegen eine Castration bei intacten Geschlechtsorganen, sondern glaubt nur, dass bei dem jetzigen Stande unsers Wissens dabei Indicationen für die Vornahme der Operation nicht begründet werden können. Wie man schliesslich Mittel und Wege finden könne, in solchen Fällen die Indication für die Castration festzustellen, habe er ja selber angegeben.

Ueber allen Zweifel erhaben sei jedenfalls ein Causalnexus zwischen nervösen Erscheinungen und nervösen Symptomencomplexen einerseits und pathologischen Processen im Sexualapparate andererseits; es sei möglich, hier durch Castration Heilung zu schaffen. Besondere Aufmerksamkeit verdiene in der Beziehung die kleincystische Degeneration der Ovarien, die bald keine Beschwerden hervorrufe, bald solche im Gefolge habe. Er habe dieselbe besonders entstehen sehen bei übermässiger geschlechtlicher Reizung. Diese führe zu excessiver Ovulation, excessiver Entwickelung zahlreicher Follikel und Störungen in der Rückbildung derselben. Dabei habe Redner hochgradige nervöse Erscheinungen bei Hysteroepilepsie, beginnende Psychose beobachtet. Wenn nun auch schwer zu entscheiden, was Ursache und Folge, so geben doch nachweisbare Veränderungen der Ovarien gute Anhaltspunkte für eine Indicationsstellung. Ausserordentlich schwierig dagegen sei es, bei bestehender Neurose eine Indication zur Castration zu begrïnden, wenn der Geschlechtsapparat gesund befunden werde. Dass Schwangerschaft, Geburt und Wochenbett Neurosen und Psychosen bedingen können, sei zweifellos; nur dürfte es schwer fallen, hier das ursächliche Moment klar zu legen, da oft andere Factoren : Gemüthsbewegungen, Heredität u.s.w. eine grosse Rolle spielen. Aehnlich verhalte es sich mit den sog. Menstrualneurosen. Es sei ihm da kein Fall bekannt, wo die 
Menstruation allein als Ursache einer grossen Neurose angesehen werden könne. Sobald Beobachtungen gemacht werden, wo bei gesundem Genitalapparate die Menstruation beschuldigt werden könne, eine starke Neurose herbeigeführt zu haben, sei er mit der Richtigkeit der Indicationsstellung zur Castration einverstanden. Die Begründung einer Indicationsstellung auf den Einfluss der Klimax auf den Organismus und das Nerrensystem sei noch schwieriger. Wir kennen als psychologische Wirkungen der Castration eine bessere Ernährung und ein ruhiges Temperament; das genügt nicht zur Aufstellung einer Anzeige. Die Bedingungen für den bald günstigen, bald ungünstigen Einfluss der natürlichen Klimax auf bestehende Neurosen und Psychosen sind uns nicht bekannt und lässt sich mithin auch damit nichts weiter anfangen.

Freund (Strassburg) erwähnt einen Fall von ovarieller Hystero-Epilepsie, wo nach Entfernung eines Dermoidkystom keine Heilung eintrat.

Olshausen ist völlig einverstanden mit den Ausführungen Schroeder's. In Bezug auf die kleincystische Degeneration der Ovarien ist er der Ansicht, dass dieselbe bald als pathologische Erscheinung aufzufassen ist, bald aber auch nicht.

Angehend die Hegar'sche Indicationsstellung weist er darauf hin, dass dieser Autor, welcher bisher fest auf anatomischem Standpunkte verharrte, doch eine wesentliche Concession macht, indem er die Berechtigung zur Castration zugiebt, wenn ein deutlicher Zusammenhang zwischen Function der Orarien und Neurose besteht, ohne dass greifbare Veränderungen am Sexualapparate vorliegen.

Schatz theilt aus seiner Praxis folgende Fälle mit, welche bewoisen, wie vorsichtig man in der Indicationsstellung sein muss.

In dem ersten Falle, wo während der Menstruation Hysteroepilepsie bestand, wurde durch Entfernung der dermoid entarteten Ovarien während fünf Jahren Heilung geschaffen. Nach typhösem Fieber kehrten die Anfälle wieder.

Im zweiten Falle wurde castrirt wegen Hysteroepilepsie im Zusammenhange mit der Menstruation. Die Ovarien waren gesund. Nachher schwanden die Anfälle fast ganz; statt der nunmehr verschwundenen Menstruation tritt jetzt seit vier Jahren ein urticariaartiger Ausschlag ein.

Im dritten Falle, wo eine Operation nicht stattgefunden hat, 
treten die Anfälle stets ein, wenn die Menstruation ausbleibt, aber nie, wenn die Menstruation auf mechanischem oder medicamentösem Wege herbeigeführt wird.

Landau hält mit Schroeder dafür, dass nicht Theorie, sondern Erfahrung uns vorläufig bestimmen müssen, bei schweren Neurosen zu castriren und sei es wohl vor der Hand gestattet, letztere Operation dann vorzunehmen, wenn alle anderen Maassnahmen erschöpft sind. Nach den von ihm mit Remak gesammelten Erfahrungen, die sich seit den bereits veröffentlichten auf 3 weitere Fälle beziehen, wo ebenfalls die Castration nicht im Stande war, die Schmerzen zu heben, ist er der Ansicht, dass man bei schweren Neurosen von der Castration Abstand nehmen soll, wenn Ovarie besteht. Denn diese ist nicht das Symptom einer Parenchym-, sondern einer centralen Erkrankung.

Gusserow steht ganz auf dem Standpunkte Schroeder's und Olshausen's. Der Hauptwerth der heutigen Verhandlung liegt nach ihm darin, dass durch die Beobachtungen Schram 's und Schroeder's festgestellt ist, dass schwere Neurosen (Epilepsie u.s.w.) durch die Entfernung beider gesunder Ovarien geheilt werden können. Dagegen können Beobachtungen, wie sie Freund anführt, nicht in Betracht kommen, denn dass bei jedem epileptischen Individuum (Mann oder Weib) Tumoren vorkommen, deren Entfernung keinen Einfluss auf die Epilepsie hat, ist eine belsannte Thatsache. Es scheint aber von Wichtigkeit zu betonen, dass an und für sich die Epilepsie eine so wenig erkannte Krankheit ist, deren Ursachen gewiss so mannigfache sind, dass wir uns vor der Vorstellung hüten müssen, als ob die Epilepsie in einem besonderen Zusammenhange mit der Ovarie stände.

Sän ger berichtet über einen Fall von schwerer Menstruationsneurose, welche zur Castration führte. Die bestehenden Schmerzen schwanden nach der Operation nur links, rechts kehrten sie wieder und wurden auf Zerrung durch Adhäsionen zurückgeführt. Nach verschiedenen anderen Maassnahmen wurde in der Folge eine zweite Laparatomie vorgenommen, bei welcher die Adhäsionen zerrissen wurden. Indessen bildeten sich diese wieder und traten damit auch die Schmerzen, wenn auch schwächer, wieder ein. Die früher vorhandenen klonischen Zuckungen dagegen blieben von der ersten Operation an dauernd weg. - Die Ovarien erwiesen sich normal; der Uterus enthielt einige Myome.

Im Weiteren erwähnt Sänger der von Flechsig beschrie- 
338 Verhandlungen der gynäkologischen Section der 59, Versammlung

benen Fälle, wo nach der Castration dauernde Heilung eintrat. Das eine Mal handelte es sich um Hysteroepilepsie, das andere Mal um Geisteskrankheit.

Schroeder: Da die Ovaralgie eine centrale Erkrankung ist, kann man nicht hoffen, sie durch Beseitigung des neuralgischen Ovarium zu beseitigen. $\mathrm{Ob}$ dies geschieht, wenn man beide Ovarien entfernt, ist nicht bekannt.

Hegar bemerkt in Bezug auf die Einwände Gusserow's und Sänger's, dass er die Castration nicht bei gesunden Eierstöcken, nur bei gesundem Genitalapparate für contraindicirt hält. In den Sänger'schen Fällen seien Anomalien, wenn auch nicht der Ovarien, so doch in anderen Theilen der Sexualorgane vorhanden gewesen. Olshausen gegenüber erinnert Hegar daran, dass die Amerikaner besonders sogar castrirt haben bei schweren Neurosen, ohne nur irgend einen Connex mit den Geschlechtsorganen oder deren Thätigkeit festgestellt zu haben.

Freund hebt den Unterschied zwischen seinem Falle und dem von Gusserow erwähnten epileptischen Manne mit einem Lipom an der Schulter hervor.

Gusserow erwidert, dass er Hegar's Anschaung wohl verstanden, allein gerade habe betonen wollen, dass durch die Beobachtungen Schroeder's und Schramm's festgestellt ist, dass die Entfernung gesunder Ovarien bei gesundem Genitaltractus schwere Neurosen heilen kann. Gegenüber Fre und bezweifelt er nicht, dass die Entfernung des erkrankten Ovarium gerechtfertigt war, allein derartige negative Erfahrungen können nicht gegen die von Schroeder und Schramm gemachten Beobachtungen verwerthet werden.

Schramm hebt in Bezug auf den ersten seiner Fälle hervor, dass die epileptischen Anfälle mit der ersten Menstruation sich einstellten, als der Genitalapparat noch gesund war. Die später sich entwickelnde Retroflexion konnte also mit der Epilepsie nicht im Zusammenhang stehen. Weitgehende Schlïsse möchte er überhaupt aus seinen Fällen nicht gezogen wissen.

Schroeder schlägt vor, für die Entfernung eines kranken, nicht stark vergrösserten Ovarium die Bezeichnung Oophorectomie und nicht $\mathrm{Ca}$ stration zu wählen.

Hegar hält eine Aenderung einer bestehenden Nomenclatur für bedenklich. Es müsse berücksichtigt werden, dass Chirurgen 
und Thierärzte sowohl die Exstirpation gesunder als kranker Eierstöcke mit dem Namen der Castration belegen.

14) Sänger (Leipzig): Zur Technik der Amputatio uteri myomatosi supravaginalis.

Der Vortragende beschreibt als intraperitoneale $\mathrm{Ab}$ kapselung ein Operationsverfahren, welches bei einem Falle eines subserös und intraligamentär entwickelten Tumor mit bestem Erfolge in Anwendung kam und welches darin bestand, dass die Geschwulst mit elastischer Ligatur in situ gelassen und das Peritoneum parietale der Bauchwand derart von beiden Seiten auf die Hinterwand des Stumpfes angenäht wurde, dass dadurch ein völliger Abschluss von der übrigen Bauchhöhle zu Stande kam.

Redner empfiehlt sehr die Versenkung der elastischen Stumpfligatur, welche er unter zehn Fällen supravaginaler Amputation sechs Mal in Anwendung brachte. Nur zwei Mal, wo auch ein Thiersch'scher Bleiring mit versenkt wurde, wurde dieser sammt der Ligatur später durch die Cervix ausgestossen. Der einzige Todesfall, den Vortragender erlebte, betraf eine Patientin, bei welcher vor Schluss der Bauchhöhle die elastische Ligatur entfernt wurde.

Besprechung.

Veit hält es für zweckmässiger, den Versuch zu machen, auch derartige schwere Fälle mit den Grundsätzen der intraperitonealen Versorgung in Einklang zu bringen. Er nimmt ferner Veranlassung zur Versorgung des Stieles Juniperusöl-Catgut auf das Wärmste zu empfehlen, das sich vollständig bewährt hat. Gegen die Versenkung von Gummiligaturen führt er zwei Erfahrungen an, in denen Drainröhren aus der Bauchhöhle in die Blase bezw. Scheide durchdrangen. In vielen Fällen mag die elastische Ligatur unbemerkt durch den After abgehen.

Fritsch betrachtet die elastische Ligatur als nothwendiges Uebel und hat ebenfalls fast immer eine spätere Ausstossung derselben erlebt.

III. Sitznngstag: Mittwoch, den 22. September.

15) Schultze (Jena): Amputation des Corpus uteri mittels Laparatomie wegen Retention der Placenta und puerperaler Sepsis. 
340 Verhandlungen der gynäkologischen Section der 59. Versammlung

Bei einer 21 Jahre alten Person, welche am 7. September eine Fehlgeburt im siebenten Monate durchmachte, gelang es wegen Enge der Cervix nicht zur Placenta zu gelangen. Uterus im Fundus getheilt. In der Folge stellten sich Zeichen von Sepsis unter Temperatursteigerung bis über $40^{\circ}$ ein. Infolge dessen wurde am 13. September zur supravaginalen Amputation des Uterus geschritten, dadurch dem septischen Processe Einhalt gethan und völlige Heilung erzielt. Redner spricht den Wunsch aus, dass es gelingen möchte, durch die genannte Indication und Operation die eine und die andere Wöchnerin, die sonst an Puerperalfieber zu Grunde ginge, zu retten.

Besprechung.

Hegar berichtet über einen Fall von Sectio caesarea bei einer seit fünf Tagen kreissenden Frau. Das Fruchtwasser war vier Tage vor der Operation abgeflossen; Muttermund für zwei Finger durchgängig, Cervicalcanal etwa $8 \mathrm{~cm}$ lang. Tetanus uteri. Uebelriechender Ausfluss. Querlage. - Der Uterus war ausserdem prolabirt, das Collum elongirt. Die Wendung unmöglich. - Der anfangs ungünstige Verlauf wurde sofort gut, als durch Irrigationen den Lochien genügender Abfluss verschafft worden war.

16) Löhlein (Berlin): Indication zur künstlichen Frühgeburt bei inneren Krankheiten.

Redner unterscheidet zwei Gruppen von Krankheiten, welche die künstliche Frühgeburt bedingen. Die erste Gruppe umfasst jene Affectionen, welche, wie Chorea gravidarum, Vomitus gravid. u. s. w., direct durch die Schwangerschaft entstehen. In Bezug auf das unstillbare Erbrechen wird vor allzurascher Einleitung der Frühgeburt gewarnt und empfiehlt Löhlein consequente Darreichung von Nutrientien und Stimulantien per rectum.

Die zweite Gruppe enthält die grosse Schaar von Krankheiten, welche mehr oder weniger zufällig mit der Gravidität combinirt sind und zu Zuständen führen können, die möglicherweise mit Unterbrechung der Schwangerschaft aufhören. Eine Zwischenstellung zwischen der ersten und zweiten Gruppe bildet die Nephritis, bei welcher die Frühgeburt indicirt sein kann wegen quälender Oedeme und Transsudate; unter gewissen Bedingungen behufs Verhütung der Eklampsie; ferner in der Absicht die Frucht zu erhalten in Fällen, wo sich die Neigung zu subplacentalen Blutungen in früherer Schwangerschaft herausgestellt hat; endlich um einen Uebergang in Nephritis vera zu verhüten. 
17) Schlesinger (Wien): Ueber operative Eingriffe bei congenitalem Mangel der Vagina.

Redner illustrirt die Frage, ob bei Atresie oder Mangel der Vagina behufs Herstellung der Impotentia cohabitandi oder behufs Beseitigung nervöser und psychischer Zustände ein operativer Eingriff erlaubt sei, durch ein diesbezügliches Beispiel. Bei einem 25jährigen Mädchen mit völlig weiblichem Typus und normalen äusseren Genitalien fand Schlesinger einen Mangel der Vagina, wahrscheinlich auch des Uterus und der Ovarien. Patientin wollte sich verheirathen, war aber so hochgradig psychisch erregt, dass sie sich mit Selbstmordgedanken befasste. Auf operativem Wege, durch schneidende Instrumente und stumpfe Gewalt schuf Schlesinger bei der Patientin eine $6-7 \mathrm{~cm}$ lange Scheide. Anfangs wurde der Canal durch Jodoformgaze, später durch Dilatatorien offen gehalten. Allmälig fing sich der Canal wieder an zu verengen und zu verkürzen. Die Heirath kam nicht zu Stande; dagegen sind die psychischen Erscheinungen bis jetzt nicht wiedergekehrt.

Besprechung.

Schroeder glaubt, dass auf ein dauerndes Bestehen einer solchen künstlichen Vagina nur dann gerechnet werden kann, wenn man wenigstens ihr Anfangsstïck mit Schleimhautlappen des Introitus ausfüttert.

18) Cohnstein(Berlin): Ueber den Blutdruck vor und nach der Geburt, nach gemeinschaftlich mit Zuntz angestellten Versuchen.

Die früher von Cohnstein und Zuntz aufgestellte Behauptung, dass die ersten Athemzïge des Fötus nach der Geburt sehr wahrscheinlich kein starkes Sinken des arteriellen Blutdruckes im Gefolge hätten, wurde bekanntlich von Preyer und Ziegenspeck bestritten. Directe Versuche bezweckten, die Controverse zu beseitigen. Dieselben wurden am reifen Schaffötus in der Weise vorgenommen, dass bei demselben, durch Sectio caesarea zugänglich gemacht, zuerst bei ungestörter intrauteriner Apnoe der Blutdruck bestimmt und dann fortgesetzt weiter nach Extraction des Thieres beobachtet wurde. Dabei stellte sich nun heraus, dass der Eingriff der Geburt und der beginnenden Respiration nur geringe Aenderungen des Druckes bewirkt, im allgemeinen eher etwas erhöht wurde. Der erste Athemzug hat ein kurzdauerndes Sinken des Druckes mit darauffolgender Steigerung 
342 Verhandlungen der gynäkologischen Section der 59. Versamminung

zur Folge. Am folgenden Tage ist der Druck meist nicht höher als nach den ersten Athemzügen. Für die Steigerung des Druckes nach den ersten Athemzügen ist die normale Aspiration des Placentarblutes nothwendig. Die Abnahme der Füllung der Arteriae umbilicales beruht nicht auf Erniedrigung des Blutdruckes, sondern hat ihren Grund wahrscheinlich in der Contraction der Ringmuskulatur der Nabelarterien.

19) Ruge, C. (Berlin): Ueber die Placenta.

Redner hat bei einer Frau, die zu Beginn der Geburt starb, die Placenta vom Uterus und von den Umbilicalarterien aus injicirt. Die erstere Injection füllte die mütterlichen uterinen Gefässe und zugleich die intervillösen Räume der Placenta. Ins Bereich der Untersuchung wurden gezogen die Uebergangsstellen des Blutes von der Mutter auf die Placenta. Redner fand dabei nirgends die bis jetzt beschriebenen durch die Serotina aufsteigenden Gefässe, sondern stellte fest, dass das Gewebe der Placenta in die Serotina und damit auch in die mütterlichen Gefässe hineinwächst. Diese werden durchbrochen und zum Theil durch Zotten ganz verstopft. Nirgends findet eine bis jetzt beschriebene Oeffnung der Gefässe statt. Wenn auch ein Uebertritt von mütterlichem Blute in grösseren oder kleineren Mengen in den intervillösen Raum nicht geleugnet werden kann, besteht doch keine intervillöse Circulation und sind die diesbezüglichen Schemata falsch.

Besprechung.

Küstner (Jena): Die intervillösen Räume, die wirklich bestehen, sind in den Schemata allerdings zu gross gezeichnet im Vergleich zu dem von den Zotten beanspruchten Platze, allein es geschieht das im Interesse der besseren Verständlichkeit und Anschaulichkeit.

Ruge betont, dass die Schemata auf einer unrichtigen Vorstellung über die interplacentare Circulation beruhen.

IV. Sitzungstag: Donnerstag, den 23. September.

20) Stratz (Berlin): Zur Lage des Uterus. Verwerthung von Leichenbefunden zur Bestimmung derselben.

Zum Zustandekommen der wichtigsten Leichenerseheinungen wirken folgende Momente: 1) die intraabdominellen Druckschwankungen nach dem Tode; erst Herabsetzung des Druckes durch 
Wegfall der Athembewegungen und des Muskeltonus, dann Steigerung durch Todtenstarre und Gasentwicklung in den Gedärmen; 2) Erschlaffung der muskulären und elastischen Elemente; 3) Aufhören der Circulation; 4) Rückenlage der Leiche. Die wichtigsten daraus sich ergebenden Leichenerscheinungen sind: 1) Schlaffheit des Uterus und seiner Anhänge; 2) Retroposition des Uterus; 3) Tiefertreten des Beckenbodens und seiner Weichtheile; 4) Klaffen des Anus; 5) trichterförmiges Klaffen des Introitus vaginae und der Urethra. Redner hat in einer Reihe von Fällen vor und nach dem Tode den Genitalbefund notirt und die eintretenden Veränderungen beobachtet. Das Nachhintenwandern des Uteruskörpers hatte in einem Falle bereits zwei Stunden nach dem Tode begonnen, war in einem anderen Falle nach drei Stunden schon vollendet.

Dass der Beckenboden tiefer tritt, kann nicht direct beobachtet werden, wird vielmehr daraus geschlossen, dass bei sämmtlichen Durchschnitten gefrorener ganzer Leichen der Beckenboden tiefer steht, als es nach den Messungen von Schroeder und Schultze an Lebenden der Fall ist.

Auf sämmtlichen schematischen Zeichnungen ist die Gestalt des Rectum falsch dargestellt; es fehlt der schliessende Sphincter externus, ferner die durch den Sphincter ani tertius bedingte Einschnürung des Rectum weiter oben.

Die Blase zeigt, wenn median aufgeschnitten, eine dreizipfelige, wenn seitlich angeschnitten eine mehr citronenförmige Gestalt. Die Wirkung der einzelnen Factoren zum Zustandekommen der Leichenerscheinungen lässt sich erkennen aus den Präparaten, bei welchen der eine oder der andere Factor vermieden worden ist. So ist z. B. bei injicirten Präparaten der Uterus stets anteflectirt, praller, grösser. Bei Vermeidung der Rückenlage sinkt auch der Uterus nicht nach hinten über.

Um möglichst vitale Verbältnisse zu schaffen, müsste ein Cadaver unmittelbar nach dem Tode in aufrechter Stellung injicirt und zur Gefrierung gebracht werden; es müssten dabei Oeffnungen in Bauchwand und Därmen angebracht werden, welche die Gasentweichung ermöglichen und eine Drucksteigerung im Abdomen vermeiden. Selbstverständlich lässt sich dabei allerdings die Schlaffheit der muskulären und elastischen Elemente nicht vermeiden. Es muss aber darauf hingewiesen werden, dass die durch den Wegfall dieser Momente bedingte Verschiebung Ikeine allzugrosse ist. Vortragender zieht mediane Gefrierschnitte allen 
344 Verhandlungen der gynäkologischen Section der 59. Versamminng

anderen Darstellungsmethoden vor. Den Schluss des Vortrages bildet die Demonstration zweier von Waldeyer herstammender Präparate.

Besprechung.

Fürst empfiehlt behufs Vermeidung von Resultaten, bedingt durch Rückenlage, Versuche an der Leiche derart anzustellen, dass man dieselbe in vertikaler Suspension mit leicht auf dem Erdboden aufruhenden Füssen studirt. Ein Abguss bei eröffneter Bauchhöhle an einer jugendlichen Person vorgenommen, ergab normale Anteflexionsstellung des Uterus. Wenn man die Versuche bald nach dem Tode, vor Eintritt der Leichenerscheinungen vornimmt, erhält man annähernd vitale Verhältnisse.

Küstner weist darauf hin, dass auch er ausführlichst betont hat, dass Leichenbefunde nicht verwerthet werden dürfen für die Kenntnisse von der Lage der Beckeneingeweide an der Lebenden.

Landau lenkt die Aufmerksamkeit der Fachgenossen auf die Rechtslagerung des schwangeren Uterus, für welche bis jetzt eine annehmbare Erklärung fehlt. Die Erklärung Landau's geht dahin, dass, weil das Mesenterium schräg von der linken Seite des ersten Lendenwirbels bis zur rechten Sacroiliacaljunctur zieht und eine relative Kürze hat, der Uterus von dem mehr links festgehaltenen Dünndarme gezwungen wird, nach rechts hin sich zu entfalten.

Hegar weist darauf hin, dass die Ansicht der Anatomen von der Lage des Uterus zum Theil darauf beruht, dass sie Leichen secirten, welche schon während des Lebens eine Retroversion besassen. Er sagt ferner, dass es Anatomen giebt, welche die Existenz des Ligamentum sacrouterinum leugnen; wohl deshalb, weil dieselben schon oft bei der Lebenden sehr atrophisch angetroffen werden, dass man sie nach dem Tode völlig übersieht.

Endlich glaubt $\mathrm{Hegar}$, dass eine Reihe von Factoren, welche nach dem Tode Retroversion bedingen, schon während des Lebens vorhanden und diese Lageanomalie herbeiführen. Erschlaffung der Bauchdecken, des Beckenbodens, der Ligamenta sacrouterina u. s. w.

Schatz ist mit der Landau'schen Erklärung der Rechtslagerung des Uterus im Allgemeinen einverstanden, möchte aber den Einfluss von der Beweglichkeit und Befestigung der Unter- 
leibsorgane auf die Stellung des Uterus noch verallgemeinert wissen.

Stratz betont mit Rücksicht auf die Ausführungen von Fürst, dass bei uneröffneter Bauchhöhle der Abdominaldruck stärker wird als unter normalen Verhältnissen.

Hegar's Ansicht stimmt er bei, glaubt aber doch durch directe Beobachtung den Beweis erbracht zu haben, dass der Uterus in der Leiche nach hinten sinkt.

21) Cohn (Berlin): Ueber die Ophthalmoblennorrhoe der Neugeborenen.

Cohn hat die Credé'schen Argentum nitricum-, die Sublimat-, die Zincum-sulfo-carbolicum-Einträufelungen verglichen mit den Resultaten, welche man erreicht bei strenger Antisepsis während der Geburt, verbunden mit sauberem Abwischen der Lider u. s. w., und kommt durch diesen Vergleich zu dem Schlusse, dass eine obligatorische Prophylaxe überflüssig sei.

Neben der ächten Blennorrhoe hat Cohn eine gutartige Conjunctivitis, nicht auf bakterischer Grundlage beruhend, gefunden.

Besprechung.

Hegar glaubt ebenfalls, dass durch blosse Reinlichkeit die Blennorrhoe vermieden werden könne. So lange er in seiner Anstalt eine reinliche, tüchtige Hebamme hatte, kam jahrelang in seiner Klinik kein Fall von Blennorrhoe vor. Sobald eine neue Hebamme antrat, erschien die Affection und verschwand dann wieder, als diese besser instruirt war. Die guten Resultate Kaltenbach's mit grosser Sauberkeit und destillirtem Wasser rühren daher, dass er jene gute Hebamme nach Giessen engagirte. Aus dem allem ist auch zu entnehmen, dass die Infection nicht so leicht beim Durchtritte des Kopfes durch die Vagina stattfindet.

Frank suchte in Cöln die prophylaktischen Einträufelungen überflüssig zu machen durch folgendes Verfahren: Vulva, Damm und Oberschenkel werden mit Carbol und Seife gewaschen; die Vagina öfters unter der Geburt ausgespült mit 3 proc. Carbollösung; unmittelbar nach dem Austritte des Kopfes wird das ganze Gesicht, bevor die Lider geöffnet werden, mit Aether gewaschen behufs Entfernung der Vernix caseosa. So lange diese Manipulationen von Aerzten vorgenommen wurden, traten keine Blennorrhoeen auf; diese waren aber sofort wieder da, wenn die Hebammen diese Proceduren vornahmen. Seit der Zeit werden die 


\section{Verhandlungen der gynäkologischen Section der 59. Versammlung}

Credé'schen Einträufelungen vorgenommen; daneben selbstrerständlich die minutiöseste Reinlichkeit beobachtet.

Gusserow hat ebenfalls schon vor über Jahresfrist in der Charité Versuche darüber angestellt, ob die Einträufelungen ron 2 proc. Höllensteinlösungen nicht ersetzt werden können durch die scrupulöseste Reinlichkeit bei der Geburt und durch Einträufelungen von destillirtem Wasser. Die Resultate waren durchaus unbefriedigend; es zeigten sich sofort wieder Fälle ron Ophthalmoblennorrhoe. Er warnt daher vor derartigen Versuchen.

Schatz weist auf die Schwierigkeiten der Verhütung der Blennorrhoe in der Privatpaxis hin, wo Gesetze der Reinlichkeit schwerlich genügen, wo wir deshalb besonderer Maassnahmen bedürfen; denn wenn auch die Blennorrhoeen in der Privatpraxis selten sind, so kommen doch gerade da relativ viele Erblindungen vor.

Korn hat in 100 Fällen die Kaltenbach'sche Prophylaxe angewandt und dabei sechs Blennorrhoeen erlebt. Seit dann die Credé 'sche Methode wieder in Anwendung kam, verschwand die Affection. In Bezug auf die Häufigkeit der Blennorrhoeen in der Privatpraxis verweist er auf die ron Prochownick ausgerèchnete Zahl von 10 bis 12 Proc. Cohn gegenüber bemerkt Korn, dass dessen Zahlen zu klein seien, um daraus weitgehende Schlüsse zu ziehen. Er habe im Beginne seiner Beobachtungen ebenfalls $4 \%$ Erkrankungen erlebt.

\section{2) J. Veit (Berlin): Ueber Endometritis corporis.}

Die Lehre von der Endometritis corporis ist durch die neueren Untersuchungen in vielen Beziehungen gefördert worden, doch scheint mir, dass über gewisse Punkte eine weitere Erörterung zweckmässig ist. Ich will aus der Anatomie hier nur einen Punkt, der mir besonders nach den Untersuchungen Wyder's über das Endometrium bei Myomen wichtig scheint, hervorheben. Derselbe betonte, dass, je näher ein Fibromyom der Schleimhaut sitzt, desto eher handele es sich um eine interstitielle Entzündung derselben. Es folgte für Wyder aus dem weiteren Umstande, dass er bei subperitonealen Myomen glanduläre Endometritis fand, dass die Blutungen wesentlich von der interstitiellen Entzïndung abhingen. Der Schluss liegt ja nun nahe, dass überhaupt die Blutungen bei Endometritis allein abhängig sind von der interstitiellen Form. Ich habe gerade seit der Mittheilung der Resultate Wyder's. mich über diesen Punkt zu orientiren versucht und ich muss 
sagen, dass ich mich bei der myomlosen Endometritis nicht habe davon überzeugen können, dass iberhaupt für die Symptome ein wesentlicher Einfluss von der anatomischen Form der Entzündung ausgeübt werden kann. Sie können das daraus schliessen, dass z. B. Schroeder die interstitielle Endometritis als Ursache der dysmenorrhoischen Form, Wyder dieselbe als Ursache der Blutungen ansieht.

Von den Punkten dieses Gebietes, die einer weiteren Discussion noch bedürfen, hebe ich in erster Linie die Diagnostik hervor. Durch bestimmte Symptome - Blutungen, Ausfluss und heftige Schmerzen - wird man veranlasst, auf das Endometrium zu achten.

Das Hauptmittel der Diagnose bleibt meiner Ansicht nach die Sonde. Ich habe zwar vielfach versucht, mich mit dem Probetampon Schultze's zu üben, bin aber nicht im Stande gewesen, mit einiger Sicherheit aus diesem allein Schlüsse darüber zu ziehen, ob ein bestehender Katarrh in der Cervix oder im Corpus sitzt. Wichtig bleibt allerdings hierfür das Ausschliessen der Betheiligung der Cervicalschleimhaut durch die Besichtigung des blosliegenden Theiles und durch Sondirung u. s. w. der höher liegenden Theile derselben. Aber ron entscheidender Bedeutung ist allein die Sonde. Mit ihr fühlt man bei den Formen, welche zu Wucherungen der Schleimhaut geführt haben, die Unebenheiten direct; mit ihr fühlt man ferner eine sehr charakteristische Weichheit der Schleimhaut. Letzteres ist allerdings nur bei vieler Uebung möglich. Endlich fühlt man mit der Sonde besondere Empindlichkeit der Uterushöhle und zwar meist an aufgeriebenen Stellen. Bei der diagnostischen Schwierigkeit, die durch die Subjectivität dieses Symptoms entsteht, scheint mir die Thatsache wichtig, dass man an den empfindlichen Stellen regelmässig entweder Unebenheiten oder abnorme Weichheit findet. Es dürfte dies wohl vor schwer wiegenden diagnostischen Irrthümern schützen.

Ein zweiter Punkt, den ich besonders hervorheben möchte, ist der Zusammenhang der Endometritis mit der Schwangerschaft. Nur allzu leicht werden die makroskopisch nicht sehr erheblichen Veränderungen übersehen; bei genauer Untersuchung stellen sie sich mikroskopisch als streifenförmige interstitielle oder mehr diffuse drüsige Wucherung in der Decidua dar. Der Grund eines Abortus ist nur durch Aufmerksamkeit hierauf zu finden - in einem Falle Amputation der Cervix wegen Carcinom in der Schwangerschaft, fieberfreie Heilung, sechs Wochen darauf Abortus, deutlich deciduale Entzündung, die wohl auf frühere Endometritis zurück- 
348 Verhandlungen der gynäkologischen Section der 59. Versammlung

zuführen war - und Heilung der „Disposition“ zum Abortus gleichfalls nur hierdurch möglich.

Endlich scheint es mir bei der Verschiedenheit der Behandlungsmethoden an der Zeit, eine genauere Kritik an die einzelnen Verfahren zu legen, und ich habe mich in dieser Hinsicht bemüht, durch den Eintritt und den normalen Verlauf von Gravidität, durch das Schwinden der Symptome und die öfter wiederholte Untersuchung kleiner Theile der Schleimhaut mehr oder weniger lange Zeit nach der Behandlung mich zu orientiren.

Einen wesentlichen Einfluss allgemeiner Therapie habe ich niemals feststellen können; vielleicht ist ein solcher vorhanden bei den selten zur Behandlung kommenden chlorotischen oder scrophulösen Formen der Erkrankung, die man bei jungen Mädchen manchmal sieht. Ebenso wenig habe ich von der sonst so viel gerühmten Behandlung mit Stäbchen, Stiften, Aetzmitteln, die auf die verschiedenste Weise eingebracht werden, viel dauernden Nutzen. gesehen. Es lässt sich eine vorübergehende, d. h. nur während der Behandlung andauernde Besserung der Symptome nicht verkennen, aber nachhaltigen Erfolg sah ich nicht; besonders mangelhaft ist diese Art der Behandlung bei der dysmenorrhoischen Form. Die Methode der Jenenser Klinik, Erweiterung mit Laminaria und Ausspülung, ist zweifellos wirksam, aber bei den einfach katarrhalischen Formen kommt man ebenso weit, wenn man bei den einfachen Formen allein, bei complicirten nach der Auskratzung energisch desinficirende Einspritzungen macht. Eine Laminariaerweiterung seheint mir nur nothwendig bei gleichzeitiger starker Verdickung der Uteruswand; gegen die hämorrhagischen Formen ist diese Methode meist nicht genügend. Hier empfehle ich Ihnen die Auskratzung, aber meist mit consequenter Nachbehandlung. Die Auskratzung allein genügt nur bei mangelhafter Rückbildung nach Abortus, ohne dass vorher eine Erkrankung der Schleimhaut etwa die Ursache des Abortes gewesen wäre; ist letzteres der Fall, so muss mehr geschehen als die einfache Auskratzung. Ebenso genügt dieselbe nur bei den leichtesten Formen der Endometritis, um eine auch anatomisch nachweisbare Heilung herbeizuführen. Auch die einmalige Einspritzung von Eisenchlorid unmittelbar nach der Auskratzung hat mich meist nicht vor Recidiven geschützt. So muss ich Ihnen für die schlimmeren Arten der Endometritis als bestes Mittel die Auskratzung mit mehrwöchentlicher Nachbehandlung der sich wieder bildenden Schleimhaut empfehlen. Unter letzterer verstehe ich bei den katarrhalischen eitrigen dysmenorrhoischen Formen die Ausspülung mit 
desinficirender Flüssigkeit, bei allen anderen Formen aber die mehrwöchentliche, alle zwei Tage sich wiederholende Einspritzung von mehreren Gramm unverdünnter Jodtinctur. So habe ich allerdings normale Schleimhaut und übrigens auch mehrfach normale Gravidität eintreten sehen.

Besprechung.

Schwarz (Halle) unterscheidet drei Formen der Endometritis:

1) Endometritis fungosa OIshausen's. Bei dieser haben Auskratzungen mit nachfolgender Injection von Liquor ferri oder Jodtinctur ausgezeichneten Erfolg. Gravidität erfolgt häufig und zwar sofort.

2) Endometritis gonorrhoica. Bei dieser fehlen. Unebenheiten und nützen Ausschabungen daher nichts. Man kommt hier zum Ziele durch Aetzmittel.

3) Endometritis senilis. Sie zeichnet sich aus durch starke Atrophie der Schleimhaut. Hochgradige Empfindlichkeit ist manchmal das einzige Symptom; zuweilen besteht auch Hypersecretion. Auch hier richtet man mit den Ausschabungen nichts aus und schreitet am besten ein mit Aetzungen, die meist rasch zu helfen pflegen, freilich zuweilen nicht für immer. Injectionen von Jodtinctur in so kurzen Zwischenpausen wie Ve it sie macht (zweitägig) hält Schwarz nicht für zweckmässig, räth dieselben höchstens alle acht Tage vorzunehmen. Man muss eben die zarte Mucosa nicht allzu sehr in die Tiefe zerstören, sondern ihr Zeit lassen sich zu erholen, wenn sie nicht allzu sehr in anatomischer und physiologischer Hinsicht geschädigt werden soll.

Fränkel (Breslau) anerlzennt das Eintheilungsprincip der verschiedenen Endometritiden von Schwarz. Für die Olshausen'sche Endometritis fungosa stimmt er der Behandlungsmethode von Veit bei mit dem Unterschiede allerdings, dass er nicht so häufig nach der Ausschabung Jodinjectionen vornimmt, wie er denn überhaupt keinen durchgreifenden Unterschied in den Heilerfolgen durch die ausschliessliche Anwendung des Jod anerkennen kann. Dasselbe wendet er besonders an bei Hypersecretion; dagegen den Liquor ferri bei vorwiegender Neigung zu Blutungen. Bei der gonorrhoischen Form sah er bei Jodinjectionen keinen besonderen Heileffect, verspricht sich da mehr von lange Zeit fortgesetzten intrauterinen Sublimatausspülungen.

Hegar hält ebenfalls die Abrasio mit zeitweisen (alle $5-6$ Tage) intrauterinen Jodinjectionen für die zweckmässigste Behandlung der Endometritis. Er hat mit der letzteren übrigens auch 
350 Verhandlungen der gynäkologischen Section der 59. Versammlung

gute Erfolge bei der gonorrhoischen Form erzielt. Zuweilen hat ihm nach Fehlschlagen anderer Mittel die Höllensteinlösung gute Dienste geleistet. Behufs Injectionen ist der Cervicalkanal weit zu machen. Es findet dann auch keine Secretstauung statt. Etwa eintretende Temperatursteigerungen belzämpft man am besten durch Irrigationen des Uterus.

Löhlein, der im Allgemeinen in Bezug auf Wesen und Bedeutung der Endometritis mit dem Vortragenden übereinstimmt, hebt hervor, dass zahlreiche Fälle, auch diffuser Endometritiden, durch die einfache Abrasio geheilt werden können. Vor allzu ausgiebiger Anwendung der Sonde behufs Auffindung schmerzhafter Stellen warnt er. Die senile Endometritis, ausser der Schmerzhaftigkeit manchmal charakterisirt durch ichorösen Ausfluss, sah Löhlein sehr schnell heilen nach Einlegung hohler intrauteriner Stifte behufs Drainage.

Schücking glaubt für die Diagnose den Schultze'schen Probetampon nicht entbehren zu können.

Küstner: Der Schultze'sche Probetampon kann insofern im Stiche lassen, als derselbe keine Antwort auf die Frage giebt, ob Cervix- oder Uteruskatarrh vorliege. Dagegen giebt er uns immer Aufschluss, ob eine quantitativ oder qualitativ pathologische Secretion besteht oder nicht. Vielleicht würde in Bezug auf ersteren Punkt der von K üstner gemachte Vorschlag, die Secrete durch verschieden gelöcherte Röhren aufzufangen, von Werth sein und es auf diese Weise gelingen festzustellen, ob bei bestehender Endometritis die pathologische Secretion sich mit ersterer deckt oder nicht.

Unzweifelhaft giebt es Endometritiden ohne Secretion. Dahin gehören die interstitiellen Formen und die von Zeller beschriebene Psoriasis uterina.

Bei einfachen nicht complicirten Endometritiden genüge zur Uleilung häufig blos die Dilatation des inneren Muttermundes und die Weiterhaltung desselben. Injectionen sind überflüssig.

Diejenigen Formen dagegen, welche mehr den Charakter einer Neubildung als einer Entzïndung tragen, behandelt man zweckmässig durch Ausschabungen.

Hirschberg reibt nach Ausschabung bei Endometritis fungosa die geschaffene Wundfläche auf der Uterusinnenwand so lange mit Jodoformgaze, bis dieselbe keine Fetzen und fungöse Reste mehr enthält. Dann folgt eine intrauterine Ausspülung. 
Nach diesem Verfahren hat Hirschberg nie Recidive auftreten sehen, obschon er nachträgliche Injectionen nicht anwendet.

\section{Beckens. \\ 23) Winter (Berlin): Zur Therapie des platten}

Vortragender hat an der Hand des poliklinischen Materiales der Königl.. Universitäts-Frauenklinik die Frage geprüft, welche der beiden Grundanschauungen in der Therapie des platten Beckens richtiger sei, die exspectative, welche mehr für spontanen Verlauf, hohen Forceps und Perforation spricht, oder die active, welche durch frühe Wendung die Entbindung abkürzt. Er entscheidet sich für die abwartende Methode bei Erstgebärenden und für die active bei Mehrgebärenden. Seine Gründe hierfür sind:

1) Bestimmte Veränderungen in der Kraft der Bauchpresse, Stärke der Wehen, Grösse des Kindskopfes, Dehnung des unteren Segmentes, welche alle einen Durchtritt des Kopfes bei Mehrgebärenden unwahrscheinlich machen.

2) Die mit diesem -Verfahren für Mutter und Kind gewonnenen äusserst günstigen Resultate.

Besprechung.

Fränkel will die Vorschläge von Winter nicht auf das allgemein verengte und allgemein verengte platte Becken übertragen wissen. Die hohe Kopfzange, als Versuch vor Perforation eines lebenden Kindes bei engem Becken, möchte or nur dem Kliniker und erfahrenen, geübten Geburtshelfer zugestehen.

Hegar ist mit der Einschränkung der prophylaktischen Wendung bei Erstgebärenden einverstanden, namentlich mit Rücksicht auf die viel grössere Wahrscheinlichkeit der Verletzungen des Dammes und der Vagina. Dagegen scheint es ihm nicht gerechtfertigt, die Gefahren bèi operativ behandelter und spontan verlaufender Geburt gleich zu setzen, da bei operativ behandelten Fällen im Allgemeinen grössere Verletzungen vorkommen und die Infectionsgefahr erhöht ist im Vergleiche mit den spontan verlaufenden Geburten. Die mitgetheilte Zusammenstellung mit den Resultaten der Spiegel berg'schen, Winckel'schen und Scanz oni'schen Kliniken ist wohl deshalb nicht völlig maassgebend, als letztere Fälle wohl noch aus der Zeit stammen, wo die antiseptischen Cautelen noch nicht streng berïcksichtigt wurden.

Schatz, der sich mit den Principien des Vortragenden im Allgemeinen einverstanden erklärt, vermisst unter der Aufzählung 
352 Verhandlungen der gynäkologischen Section der 59. Versammlung

der Factoren, welche die spontane Geburt bei Mehrgebärenden mit plattem Becken erschweren, das Fehlen genügender Steifung der Frucht, wenn der Contractionsring zu. hoch gezogen ist. Selbst bei durchaus genügendem Uterus - und Bauchpressendruck wird die Geburt dadurch erschwert, dass Fruchttheile, über dem Beckeneingange befindlich, keine genügende seitliche Compression erfahren.

Freund sieht in der relativen Indication zum Kaiserschnitte eine wichtige Bereicherung der Therapie der Geburt bei engem Becken, um so mehr, als die Sectio caesarea, nach des Sprechers Erfahrungen, eine sehr sichere Operation ist. Im Uebrigen stimmt er der von Winter gegebenen Indicationsstellung bei Erst- und Mehrgebärenden bei.

Winter erwidert Fränkel, dass seine Vorschläge nur auf das platte Becken sich beziehen; beim allgemein verengten warnt er ebenfalls vor zu häufiger Wendung wegen der durch die Verengerung im ganzen Geburtskanale sehr erschwerten Extraction des nachfolgenden Kopfes. Gegenüber Hegar betont er, dass auch er in der Gefahr der Dammrisse bei der Wendung bei Erstgebärenden einen Grund zur Beschränkung derselben sehe; einen geringen Unterschied in der Morbidität zwischen operativ und spontan beendeten Geburten zu Ungunsten der ersteren erkennt er ebenfalls an, doch hält er denselben für nicht so bedeutend, dass daraus ein Umschlag in der Fixirung der Therapie erfolgen könne. Eine Gegenüberstellung der Resultate aus den verschiedenen Kliniken hält ex deshalb nicht für so unberechtigt, weil die Winckel'schen und Scanzoni'schen erst vor drei und vier Jahren publicirt, ebenfalls zum grossen Theile der antiseptischen Zeit angehören.

24) M. Hofmeier (Berlin): Ueber die endgültige Heilung des Carcinoma cervicis uteri.

Auf Grund von 161 bis zum 1. October 1885 in der Universitäts - Frauenklinik operirten Fällen von Carcinoma cervicis (115 partielle, 46 totale Exstirpationen) erläutert Ho fmeier die Frage nach der bleibenden Wirkung der Operationen. Unter Fortlassung der an der Operation Verstorbenen oder sonst Verschollenen, hat er Jahr für Jahr den weiteren Gesundheitszustand dieser Operirten verfolgt und kommt dabei zu dem Resultate, dass, entgegengesetzt den früheren Anschauungen, die Prognose der ope- 
rativen Behandlung dieser Fälle eine sehr gute ist. Beifolgende tabellarische Uebersicht erläutert diese Anschauung:

Carcinom-0perationen von Heilungsdauer über ein Jahr vom 1. October 1878 bis dahin 1885 .

\begin{tabular}{|c|c|c|c|c|c|}
\hline $\begin{array}{l}\text { Geheilt } \\
\text { p. Operat. }\end{array}$ & Operationen. & $\begin{array}{c}\text { Gesammt- } \\
\text { zabl }\end{array}$ & Recidive. & Heilungen & Procentsatz \\
\hline \multirow[t]{2}{*}{ 1. Jahr } & $\begin{array}{c}\text { Partielle } \\
\text { Totale }\end{array}$ & $\begin{array}{l}97 \\
33\end{array}$ & $\begin{array}{l}47 \\
13\end{array}$ & $\begin{array}{l}50 \\
20\end{array}$ & $\begin{array}{l}51,5 \% \\
63,6 \%\end{array}$ \\
\hline & Summa & 130 & 60 & 70 & $54,5 \%$ \\
\hline \multirow[t]{2}{*}{ 2. Jahr } & $\begin{array}{c}\text { Partielle } \\
\text { Totale }\end{array}$ & $\begin{array}{l}85 \\
29\end{array}$ & $\begin{array}{l}45 \\
22\end{array}$ & $\begin{array}{r}40 \\
7\end{array}$ & $\begin{array}{l}47,3 \% \\
24,1 \%\end{array}$ \\
\hline & Summa & 114 & 67 & 47 & $41,2 \%$ \\
\hline \multirow[t]{2}{*}{ 3. Jahr } & $\begin{array}{c}\text { Partielle } \\
\text { Totale }\end{array}$ & $\begin{array}{l}62 \\
23\end{array}$ & $\begin{array}{l}34 \\
17\end{array}$ & $\begin{array}{r}28 \\
6\end{array}$ & $\begin{array}{l}45,2 \% \\
26 \%\end{array}$ \\
\hline & Summa & 85 & 51 & 34 & $40 \%$ \\
\hline \multirow[t]{2}{*}{ 4. Jahr } & $\begin{array}{c}\text { Partielle } \\
\text { Totale }\end{array}$ & $\begin{array}{l}49 \\
10\end{array}$ & $\begin{array}{l}28 \\
10\end{array}$ & $\begin{array}{r}21 \\
0\end{array}$ & $\begin{array}{l}43 \% \\
0 \%\end{array}$ \\
\hline & Summa & 59 & 38 & 21 & $35,5 \%$ \\
\hline \multirow[t]{2}{*}{ 5. Jahr } & $\begin{array}{c}\text { Partielle } \\
\text { Totale }\end{array}$ & 26 & 15 & 11 & $42,3 \%$ \\
\hline & Summa & & & & \\
\hline 6. Jahr & Partielle & 11 & 6 & 5 & $45 \%$ \\
\hline
\end{tabular}

An der Hand der einzelnen Krankengeschichten hat er dann die Geheilten und recidivirenden Fälle gesichtet und sucht daraus die Prognose für die einzelnen Formen des Cervixcarcinoms und für den bestimmten Fall abzuleiten und die Frage zu prüfen, ob die Totalexstirpation in allen Fällen nothwendig gewesen sei. Es wird der Schluss gezogen, dass auch jetzt für die fortgeschrittenen Fälle eine Heilung auf operativem Wege nicht zu erreichen, im früheren Stadium dagegen mit aller En ergie anzustreben ist.

Besprechung.

Freund (Strassburg) berichtet über einen Fall von Uteruscarcinom, der von ihm vor acht Jahren radikal operirt wurde und bis jetzt von Recidiv verschont geblieben ist. Im Ferneren berichtet er über seine Exstirpationen aus der neuesten Zeit. Er begrüsst die Resultate der in der Berliner Klinik des Hrn. Schroede $\boldsymbol{x}$ ernst und consequent unternommenen Arbeiten auf diesem schwierigen Gebiete.

Schatz möchte wissen, ob die Statistik $\mathrm{H}$ ofmeier's auch 
354 Verhandlungen der gynäkologischen Section der 59. Versammlung

einen Vergleich enthält zwischen den mit Messer und mit Glüheisen operirten Fällen von Portiocarcinom. Da Recidive durch Einimpfung nicht so ganz selten sind, ist die Frage berechtigt, ob der Thermocauter nicht mehr vor solchen schützt als das Messer. Als Beleg führt Schatz einen Fall ron vaginaler Totalexstirpation an, wo dreiviertel Jahre später ein Cylinderzellencarcinom in der Harnröhre auftrat und führt dieses Recidiv zurück auf Einimpfung beim Katheterismus vor der ersten Operation.

Odebrecht (Berlin): Ein hierher gehöriger Fall ist folgender: hohe Excision bei Cancroid; Spaltung des Dammes wegen Enge der Vagina; nach der Operation wird die Dammwunde durch Naht vereinigt und heilt per primam. In der Narbe entwickelte sich in unmittelbarem Anschluss ein Recidiv. Redner empfiehlt die hohe Excision, wenn möglich, vorzunehmen, aber daran Kauterisation der Wunde anzuschliessen.

Fränkel berichtet mit Rücksicht auf die Frage von Schatz über drei bis jetzt recidivfrei gebliebene Amputationen der Vaginalportion; die beiden ersten wurden mit der galvanocaustischen Schlinge vorgenommen; bei der letzten kam Messer und Scheere und nachher das Ferrum candens in Anwendung. Fränkel ist der Ansicht, dass bei Anwendung des Glüheisens die Möglichkeit einer Recidivfreiheit dadurch gegeben sei, dass dasselbe eine sehr feste Narbe setze.

Schroeder verkennt die guten Eigenschaften des Glüheisens nicht, erzählt bei der Gelegenheit einen Fall, wo er ein Recidiv nach blutiger Operation vor acht Jahren dauernd beseitigt hat. Wo es sich um radikale Beseitigung eines Carcinoms handelt, bevorzugt er den Schnitt mit nachfolgender Naht als glatte sichere Methode. Wenn auch der Fall von Odebrecht sehr für eine lokale Infection zu sprechen scheint, muss man doch vorsichtig in der Beurtheilung solcher Beobachtungen sein. Ein Mädchen, von Schroeder an einem suspecten enchondromatösen Tumor der Ovarien operirt, erkrankte, obschon sicher keine malignen Zellen mit der Wunde in Berührung gekommen waren, an einem carcinomatösen Recidiv in der Bauchnarbe. Vielleicht kommt es in Fällen von allgemeiner Carcinose besonders leicht zur Ablagerung der Keime in dem neugebildeten Gewebe der Narbe.

25) Wyder (Berlin): Ueber die Behandlung der Placenta praevia.

Vortragender hebt als Zweck seiner Rede hervor, die Vor- 
theile der combinirten Wendung bei Placenta praevia zu beleuchten und dieselbe besonders dem Praktiker warm zu empfehlen. Die Hauptnachtheile der älteren, früher allgemein, jetzt auch noch bei den meisten praktischen Aerzten üblichen Methode (Tamponade bis die innere Wendung möglich, dann Wendung, Extraction, Entfernung der Placenta) sind: 1) die Blutung wird nicht sicher gestillt und muss die Tamponade öfters erneuert werden. 2) Die Gefahr der Infection ist sehr gross. 3) Risse ins untere Uterinsegment mit consecutiver, gefährlicher, schwer stillbarer Blutung. 4) Die Placenta muss oft manuell entfernt werden. 5) Der Zeitverlust ist ein sehr grosser. Alle diese Nachtheile werden durch die combinirte Wendung auf ein Minimum gebracht. Im Anschluss an dieselbe soll nicht extrahirt, sondern die Geburt völlig der Natur überlassen werden, woraus namentlich ein grosser Vortheil für die dritte Geburtsperiode erwächst.

Die gegen die combinirte Wendung erhobenen Einwände, unter ihnen namentlich die Schwierigkeit der Ausführung und die grosse Mortalität der Kinder lassen sich leicht beseitigen. Die Operation ist, wenn die ganze Hand in die Vagina eingeführt und der oder die Finger in ihrer ganzen Länge durch den Muttermund geschoben werden, sehr leicht. Bei Placenta praevia totalis soll die Placenta ohne weiteres durchbohrt und dabei, behufs Vermeidung einer stärkeren Loslösung, der vorliegende Kindestheil durch Druck von aussen als Widerlager benutzt werden. Der Vorwurf der grossen Kindersterblichkeit trifft zu, kann aber bei einem Blicke auf eine vergleichende Statistik nicht aufrecht erhalten werden, da die combinirte Wendung bei Placenta praevia, zum Ersatz für die Kinder, die Mortalität der Mütter bedeutend. vermindert hat.

Die Mortalität der Mütter beträgt nach der älteren

Methode . bei combinirter Wendung $30-40 \%$
$7,2, \% !$

Die Sterblichkeit der Kinder wird nach ersterer auf geschätzt.

$$
\text { nach letzterer auf }
$$$$
50-75,
$$$$
65,
$$

Verwerthet wurden zu dieser Statistik der combinirten Wendung 101. Fälle aus der Schroeder'schen, 88 aus der Gusserow'schen Klinik.

B esprechung.

Jung blu th (Aachen) hebt die Vorzüge seiner Pressschwämme ${ }^{1}$ )

1) Siehe Volkmann'sche Sammlung klinischer Vorträge, Nr. 235. 
sowohl für die gynäkologische als für die geburtshülfliche Praxis hervor. Er hat dieselben bis jetzt in zehn Fällen von Placenta praevia mit durchaus günstigem Erfolge angewandt.

Hofmeier weist darauf hin, dass die combinirte Wendung keine schlechteren Resultate liefere in Bezug auf die Mortalität der Kinder. Der Hauptrortheil der ersteren Methode ist und bleibt die ausserordentlich viel bessere Prognose für die Mütter.

Jungbluth hebt als Hauptrortheil seiner Methode unter anderen hervor, dass bei derselben die Kinder deshalb weniger sterben, weil dabei die Blase bis zu völliger Erweiterung des Muttermundes erhalten bleibe.

Wyder wirft der Jungbluth'schen Behandlung der Placenta praevia folgende Nachtheile ror:

1) kann der Pressschwamm, ein glücklicherweise aus der Geburtshïlfe jetzt fast ganz verbannter Körper, leicht in den Händen vieler Aerzte als Infectionsträger wirken;

2) wird bei dieser Methode, da ja die Blase erhalten bleibt und ein Zug am unteren Eipol und damit an der Placenta fortdauert, die Loslösung der Placenta vor dem operativen Eingriffe (Wendung, Extraction) nicht vermieden;

3) Werden mit Entfernung der Pressschwämme leicht die blutstillenden Coagula wieder losgerissen und macht sich dieser Umstand hauptsächlich in der dritten Geburtsperiode durch Blutungen geltend, welche eine manuelle Lösung der Placenta bedingen, eine Operation, welche bei ihrer Gefährlichkeit so viel als möglich vermieden werden sollte. Unter sieben Fällen Jungbluth's war letzterer Eingriff vier Mal nöthig; unter 27 Fällen des Vortragenden ebenfalls nur $4 \mathrm{Mal}$. Eine Zahl von 10 Fällen, wie sie Jungbluth zur Verfügung stehen, ist überhaupt noch viel zu klein, um Vortheile vor einer anderen Methode hervortreten zu lassen.

Jungbluth sagt, dass die Herausnahme der Pressschwämme erst erfolgt, wenn sie soweit gelockert sind, dass daneben der Finger bequem passiren kann: dabei können dann aber doch keine Thromben gelöst werden. Die viermalige Lösung der Placenta erfolgte nicht Blutungen halber. Er habe ausdrücklich hervorgehoben, dass infolge der Pressschwammbehandlung Nachblutungen nicht nur gleich nach der Geburt, sondern auch später fehlten, dass in seinen zehn Fällen keine Mutter gestorben oder septisch inficirt worden sei und sich die Rückbildung des 
Uterus besonders rasch und glatt vollzogen habe. Das könne nicht blos Zufall sein, sondern sei die einfache Folge der Behandlung mit Pressschwamm. Den aseptischen Charakter seiner Pressschwämme auch in etwa 120 rein gynäkologischen Fällen werde er in einer späteren Veröffentlichung nachweisen.

26) Neugebauer jun. (Warschau): Ueber Polymastie.

Neugebauer hat einen Fall von Polymastie beobachtet bei einer II para. Ausser den zwei an normaler Stelle sitzenden Brustwarzen waren noch acht andere vorhanden, welche in zwei nach unten convergirenden Reihen symmetrisch lagen. Die beiden obersten befanden sich in der Achselhöhle. Drei Paare der überzähligen lagen oberhalb der normalen Warzen, ein Paar unterhalb, die eine etwas tiefer als die andere. Das oberste Paar hatte keinen Warzenhof, dagegen die anderen sämmtlich. Nach der ersten Geburt war die Anomalie unentdeckt geblieben; am zweiten Tage des zweiten Wochenbettes fiel es der Frau auf, dass ausser aus den normal liegenden Warzen auch aus bisher für blosse Pigmentflecken gehaltenen Gebilden Milch aussickerte. Während das Kind an der normalen Brust saugte, secernirten mehrere der anderen Warzen ebenfalls Flüssigkeit. Hauptsächlich belästigt wurde die Frau durch die axillaren Brustwarzen, welche stark nässten. Die Wöchnerin functionirt zur Zeit als Amme. Die obersten accessorischen Warzen entleeren die Milch spontan, die drei unteren Paare nur auf Druck.

(Schluss der Sitzungen.)

Nächster Versammlungsort: Wies baden.

Als Commissionsmitglieder werden gewählt Diesterweg (Wiesbaden) und Kaltenbach (Giessen). 